\title{
CHARACTERISTIC CLASSES FOR SPHERICAL FIBRATIONS WITH FIBRE-PRESERVING FREE GROUP ACTIONS
}

\author{
Benjamin M. Mann and Edward Y. Miller
}

\begin{abstract}
Let $G(H)$ be the monoid of $H$ equivariant self maps of $S(n V)$, the unit sphere of $n$ copies of a finite dimension orthogonal representation $V$ of a finite group $H$, stabilized over $n$ in an appropriate way. Let $S G(H)$ be the submonid of $G(H)$ consisting of all degree 1 maps. If $H_{1}$ is a subgroup of $H$ there is a natural forgetful map $S G(H) \rightarrow S G\left(H_{1}\right)$ and if $Z$ is the center of $H$ there is a natural action map $B Z \times S G(H) \rightarrow$ $S G(H)$ induced by the natural action of $Z$ on $H$. The main results of this paper are the calculations of the Hopf algebra structures of $H_{*}\left(S G\left(\mathbf{Z} / p^{n}\right), \mathbf{Z} / p\right)$ and $H_{*}\left(B S G\left(\mathbf{Z} / p^{n}\right), \mathbf{Z} / p\right)$ for all $n$ and all primes $p$, the calculations in homology of forgetful maps induced by the natural inclusions $\mathrm{Z} / p^{n-1} \rightarrow \mathrm{Z} / p^{n}$ and, for $H=\mathbf{Z} / 2$, the calculation of the action $\mathbf{m}$ ap $H_{*}\left(\mathbf{R} P^{\infty}, \mathbf{Z} / 2\right) \otimes H_{*}(B S G(\mathbf{Z} / 2), \mathbf{Z} / 2) \rightarrow$ $H_{*}(B S G(\mathbf{Z} / 2), \mathbf{Z} / 2)$.
\end{abstract}

Introduction. In this paper we study oriented spherical fibrations modeled on an orthogonal representation $V$ of a finite group $H$, a theory first studied by Segal [25], and Becker and Schultz [2]. More precisely we consider fibrations with a fibre-preserving action of $H$ on the total space such that the fibre is $H$ equivariantly homotopy equivalent to the unit sphere $S(V)$ of $V$. We stabilize these fibrations by forming the fibrewise join with the trivial $S(V)$ bundle. As we have specific geometric applications in mind, we concentrate on the case where $S(V)$ is $H$-free.

Well known results of Barrett, Gugenheim, and Moore [1], May [15], Stasheff [29], and Waner [35], reduce this question to the study of the homotopy-type of the classifying space of the submonoid $S G(H)$ of degree 1 maps in the space

$$
G(H)=\underset{\vec{n}}{\lim } \operatorname{Map}_{H}(S(n V), S(n V))
$$

of stable equivariant self-maps of $S(n V)$. This is the model studied by Becker and Schultz [2]. They showed for any compact Lie group $H$, that $G(H)$ is homotopy equivalent to $Q\left(B H^{\zeta}\right)$, where $Q(X)$ is the union over $n$ of $\Omega^{n} \Sigma^{n} X$, and $B H^{\zeta}$ is the Thom space of the vector bundle $\zeta$ over $B H$ associated to the adjoint representation of $H$ on its Lie algebra. Notice that if $H$ is finite then $\zeta=0$, and $B H^{\zeta}=B H^{+}$, the disjoint union of $B H$ 
with a distinguished base point. Becker and Schultz also show that $S G(H)$, the degree 1 component of $G(H)$, is, in fact, an infinite loop space under the composition product.

Of course when $H$ is the trivial group one obtains the classically important space $G$ which has been the cornerstone for much important work in the study of smooth, piecewise linear and topological manifolds. Milgram [18], computed the Hopf algebra structure, under composition product, of $H_{*}(S G, \mathbf{Z} / p)$ for all primes $p$. The Hopf algebra structure of $H_{*}(B S G, \mathbf{Z} / p)$ was obtained by Madsen [11], for the prime 2 and independently by May [6], and Tsuchiya [33], for odd primes. These computations were all obtained by relating the composition product in $S G$ to the loop sum and composition product in $Q\left(S^{0}\right) . Q\left(S^{0}\right)$ was, in turn, approximated by finite models where the loop sum and composition product were interpreted directly in terms of homomorphisms of the symmetric groups.

Additional structure is present in the equivariant case as the classifying space, $B C$, of the center $C$ of $H$ acts naturally on both $S G(H)$ and $B S G(H)$. In fact this action, when $H=\mathbf{Z} / 2$, provided the initial motivation for this paper as well as our joint work with Haynes Miller [12], and [13]. More precisely we show that when $H=\mathbf{Z} / 2$ this action is geometrically represented by the "tensoring of a real line bundle with a $\mathbf{Z} / 2$-equivariant spherical fibration." This information is used in [14], to investigate the question of which unoriented $P L$ cobordism classes admit mapping tori and projective bundle representatives.

Further additional structure comes from group inclusions $H_{1} \subset H_{2}$ which induce transfer maps $t: S G\left(H_{2}\right) \rightarrow S G\left(H_{1}\right)$ and $B t: B S G\left(H_{2}\right) \rightarrow$ $B S G\left(H_{1}\right)$ which are infinite loop maps.

The main results of this paper, which are stated in Chapter I, sections two through four, are the determination of the Hopf algebras, with respect to the composition product, of $H_{*}\left(S G\left(\mathbf{Z} / p^{n}\right), \mathbf{Z} / p\right)$ and $H_{*}\left(B S G\left(\mathbf{Z} / p^{n}\right), \mathbf{Z} / p\right)$ for all primes $p$, the determination of the action $\operatorname{maps} H_{*}(B \mathbf{Z} / 2, \mathbf{Z} / 2) \otimes H_{*}(S G(\mathbf{Z} / 2), \mathbf{Z} / 2) \rightarrow H_{*}(S G(\mathbf{Z} / 2), \mathbf{Z} / 2)$ and $H_{*}(B \mathbf{Z} / 2, \mathbf{Z} / 2) \otimes H_{*}(B S G(\mathbf{Z} / 2), \mathbf{Z} / 2) \rightarrow H_{*}(B S G(\mathbf{Z} / 2), \mathbf{Z} / 2)$ and the determination of the transfer maps in homology associated to the inclusions of $1 \subset \mathbf{Z} / p^{n-1} \subset \mathbf{Z} / p^{n}$. We remark that in a closely related paper with Haynes Miller [13], we computed $H_{*}\left(S G\left(S^{1}\right), \mathbf{Z} / p\right)$ and $H_{*}\left(B S G\left(S^{1}\right), \mathbf{Z} / p\right)$ as well as the transfer maps associated to the inclusions $\mathbf{Z} / p^{n} \subset S^{1}$, and obtained results quite similar in spirit to those obtained here. 
Our study of $G(H)$ began because we were interested in computing characteristic classes for certain natural $P L$ constructions. Hence before explaining how we prove the results of this paper we briefly state, without proof, some relevant geometric facts that led us to study $G(H)$. The construction of geometric representatives for generators for $P L$ bordism (see [14]) should (but does not at present) procede by means of projective bundles as it does in the smooth category. Moreover, finite group actions on $P L$ manifolds should have the same computational characteristic class underpinnings that smooth actions do. In the semi-free setting the relevant bundle classifying spaces may be described as follows.

First there exist equivariant classifying spaces for stable piecewise linear and topological block bundles which admit block preserving $H$ actions which are free off the zero section. Further there are natural inclusions $B S O \rightarrow B S P L(H) \rightarrow B S T O P(H) \rightarrow B S G(H)$ with the evident fibres $G / P L(H), G / T O P(H)$ and so on. Secondly there are natural action maps $B C \times B S P L(H) \rightarrow B S P L(H)$ and $B C \times B S T O P(H) \rightarrow$ $B S T O P(H)$ obtained by twisting by the center which are compatible with the natural inclusions mentioned above. For example when $H=C=$ $\mathbf{Z} / 2\left(S^{1}\right)$ the action map on the $B S O(B U)$ level classifies tensor product with a real (complex) line bundle. Thirdly there are unstable versions of points one and two.

It is evident that the results of this paper play the same role in the equivariant category as the results of [18], [11], [6] and [33] on $H_{*}(G)$ and $H_{*}(B G)$ played in the fundamental calculations of Brumfiel, Madsen, and Milgram [5], of $H_{*}(B P L)$ and $P L$ cobordism. In future papers we intend to obtain calculational control of $B S P L(H), B S T O P(H)$, their associated action maps and investigate the geometric consequences of these calculations.

To prove our results we follow the same general procedure used in the classical, nonequivariant, case. In Chapters II, III, and IV we prove the theorems necessary to reduce all computations of homology groups, Pontrjagin products, homology operations, action maps and transfer maps for $S G(H)$ and $B S G(H)$ to computations in certain finite models. Before explaining this reduction, we mention that in our companion paper with Haynes Miller [12], we carry out the computations in the correct finite models which yield the results stated in Chapter I. The computations are described in Chapter V. 
The methods we use apply the $E_{\infty}$-ring space machinery of May [16], and [17]. We take this opportunity to point out that current work of Hauschild, May, and Waner [9], on equivariant infinite loop space theory gives the general $E_{\infty}$-ring space theory for based equivariant spaces. Their machinery is in a setting of much greater generality than the free and semi-free cases we consider here. We do give a complete description of the machinery necessary to make all our required homological calculations. This is done not only for the convenience of the reader but also in order to identify the free model and to describe the action of the center which has important geometrical consequences (see Chapter IV and [14]).

To explain the reduction of our problem to finite models, we begin by considering a space studied by Segal [25], [27]. Segal examined

$$
F(H)=\underset{m, n}{\lim } \operatorname{Map}_{H}\left(t\left(m V \oplus R^{n}\right), t\left(m V \oplus R^{n}\right)\right)
$$

the space of based equivariant self-maps of the one point compactification $t\left(m V \oplus R^{n}\right)$ of $m V \oplus R^{n}$ stabilized by forming the limit over equivariant inclusions of orthogonal representations. Inclusion and radical extension gives a map from the Becker-Schultz space to the Segal space $\rho: G(H) \rightarrow$ $F(H)$. Unlike the classical case ( $H=$ id), in general $G(H)$ does not fill out a component of $F(H)$. Nonetheless, $\rho$ is an infinite loop map, with respect to the composition product, and in Chapter III we express $G(H)$ as a factor of $F(H)$.

Segal also considered the semi-free Burnside space of $H, S A(H)$, a group completion of the monoid structure induced by disjoint union on the classifying space of a small category, $S Q(H)$, equivalent to the category of finite $H$ sets and their equivariant automorphisms. In fact Segal constructed a natural configuration map $\phi: B S Q(H) \rightarrow F(H)$ and thereby obtained the decomposition $F(H) \simeq Q\left(S^{0}\right) \times Q\left(B H^{+}\right)$as spaces.

We next require a result of Hauschild, May and Waner [9], which using another model for $B S Q(H), K^{H}$, considers the $E_{\infty}$-ring compatibility of the Segal decomposition. More precisely, in Chapter II we consider the $E_{\infty}$ operad pair $\left(\mathscr{K}^{H}, \mathcal{L}^{H}\right)$ discovered by Steiner [30], and show it acts on $K^{H}$ and $F(H)$ so as to make both space $E_{\infty}$-ring spaces and $\phi$ an $E_{\infty}$-ring map. Independently Segal [27], and Hauschild [8], have shown $\phi$ is a group completion. The $\mathcal{L}^{H}$ structure on $F(H)$ and $G(H)$ correspond to the composition product. 
Thus to obtain the desired results concerning $S G(H)$, we need only compute the $\mathcal{L}^{H}$ product structure on the correct factor of $K^{H}$. We isolate this factor in Chapter III and the Appendix.

In Chapter IV we define the center action map for all finite groups $H$. We then restrict our attention to $\mathbf{Z} / 2$ where we prove the homological action map is the same as the geometric twist map of [14] and $\S 4.3$.

Finally we note that there are more general versions of based equivariant function spaces, where the $H$ action is not assumed to be semi-free, and that many of our results, especially $2.1,2.2$, and 4.1, hold for these more general spaces with no essential change in the proofs. In particular, the center action map is well-defined for all these spaces and homologically described in [12].

We would like to thank Jim Becker, Peter May, Jim Milgram, Reinhard Schultz, Graeme Segal, Bob Thomason, and, most importantly, our coworker Haynes Miller, for helpful correspondence, discussions, and comments during the preparation of this paper.

\section{ChAPTER I}

1.1. In this section we define the Becker-Schultz and Segal equivariant function spaces, related transfer maps and the $\mathbf{Z} / 2$ and $S^{1}$ equivariant $J$ homomorphisms which are our fundamental objects of interest.

Let $H$ be a finite group and let $V$ be a fixed even dimensional real representation of $H$ such that $H$ acts freely on $V-\{0\}$. Further, suppose $V$ has an $H$ invariant metric. Let $\mathbf{R}$ be the trivial 1-dimensional real representation of $H$.

Definition 1.1.1. $G(H)=\lim _{\rightarrow} \operatorname{Map}_{H}[S(n V), S(n V)]$, is the space of $H$-equivariant self-maps of the unit sphere $S(n V)$ of $n V$ stabilized.

Definition 1.1.2. $F(H)=\lim _{\rightarrow, n}\left[t\left(m V \oplus \mathbf{R}^{n}\right), t\left(m V \oplus \mathbf{R}^{n}\right)\right]$ is the space of $H$-equivariant pointed self-maps of the one point compactification $t\left(m V \oplus \mathbf{R}^{n}\right)$ of $\left(m V \oplus \mathbf{R}^{n}\right)$ stabilized.

REMARKS.

1. $S(n V)$ is $H$-free.

2. To form the limits above one uses the suspension maps $\sigma_{G}\left(n_{1}, n_{2}\right): \operatorname{Map}_{H}\left(S\left(n_{1} V\right), S\left(n_{1} V\right)\right) \rightarrow \operatorname{Map}_{H}\left(S\left(n_{2} V\right), S\left(n_{2} V\right)\right)$ 
and

$$
\begin{gathered}
\sigma_{F}\left(m_{1}, n_{1}, m_{2}, n_{2}\right): \operatorname{Map}_{H}\left(t\left(m_{1} \oplus \mathbf{R}^{n_{1}}\right), t\left(m_{1} V \oplus \mathbf{R}^{n_{1}}\right)\right) \\
\rightarrow \operatorname{Map}_{H}\left(t\left(m_{2} V \oplus \mathbf{R}^{n_{2}}\right), t\left(m_{2} V \oplus \mathbf{R}^{n_{2}}\right)\right)
\end{gathered}
$$

defined by the formula

$$
\begin{gathered}
\sigma_{G}\left(n_{1}, n_{2}\right) f=f \wedge \mathrm{id}_{\left(n_{2}-n_{1}\right) V}, \\
\sigma_{F}\left(m_{1}, n_{1}, m_{2}, n_{2}\right) f=f \wedge \operatorname{id}_{\left(m_{2}-m_{1}\right) V \oplus\left(n_{2}-n_{1}\right) \mathbf{R}}
\end{gathered}
$$

where we use the $H$-equivariant homeomorphisms and identifications

$$
S\left(n_{1} V\right) \wedge S\left(\left(n_{2}-n_{1}\right) V\right) \cong S\left(n_{2} v\right)
$$

and

$$
t\left(m_{1} V \oplus \mathbf{R}^{n_{1}}\right) \wedge t\left(\left(m_{2}-m_{1}\right) V \oplus \mathbf{R}^{n_{2}-n_{1}}\right) \cong t\left(m_{2} V \oplus \mathbf{R}^{n_{2}}\right)
$$

which follows as $V$ has an $H$ invariant metric.

$G(H)$ and $F(H)$ were studied by Becker and Schultz [2], and Segal [25], respectively. Composition of maps gives each space an infinite loop space structure and the natural map

$$
\rho: G(H) \rightarrow F(H)
$$

induced by the inclusion and radial extension from $S(m V)$ to $t\left(m V \oplus \mathbf{R}^{n}\right)$ is an infinite loop space map with respect to the composition product (see 2.2.7 and 2.3.1).

Let $H_{1} \hookrightarrow H_{2}$ be an inclusion of a subgroup. Representations of $H_{2}$ restrict to give representations of $H_{1}$ and any $H_{2}$ equivariant map $f$ : $S\left(n V_{H_{2}}\right) \rightarrow S\left(n V_{H_{2}}\right)$ or $f: t\left(m V_{H_{2}} \oplus \mathbf{R}^{n}\right) \rightarrow t\left(m V_{H_{2}} \oplus \mathbf{R}^{n}\right)$ is trivially $H_{1}$ equivalent. Thus we obtain the canonical "forgetful maps"

$$
\begin{aligned}
& t: G\left(H_{2}\right) \rightarrow G\left(H_{1}\right), \\
& t: F\left(H_{2}\right) \rightarrow F\left(H_{1}\right)
\end{aligned}
$$

which are also infinite loop space maps with respect to the composition product.

We note that Segal and Becker and Schultz showed $F(H)$ and $G(H)$ are homotopy equivalent to $Q\left(S^{0}\right) \times Q\left(B H^{+}\right)$and $Q\left(B H^{+}\right)$respectively and furthermore, they identified the forgetful maps with the associated transfer maps. The following commutative diagrams summarize the situation. 


$$
G\left(\mathrm{H}_{2}\right) \stackrel{\sim}{\rightarrow} Q\left(\mathrm{BH}_{2}^{+}\right)
$$

$$
\begin{aligned}
& \downarrow \text { forget } \quad \downarrow t \\
& G\left(H_{1}\right) \stackrel{\sim}{\rightarrow} Q\left(B H_{1}^{+}\right)
\end{aligned}
$$

and

$$
\begin{array}{ccc}
F\left(H_{2}\right) & \stackrel{\sim}{ } & Q S^{0} \times Q\left(B H_{2}^{+}\right) \\
\downarrow \text { forget } & & \downarrow t \\
F\left(H_{1}\right) & \sim & \\
& \rightarrow & S^{0} \times Q\left(B H_{1}^{+}\right) .
\end{array}
$$

The precise relation between 1.1.5 and 1.1.6 is given in Chapter III.

We are primarily interested in the +1 component of $G(H)$ which we call $S G(H)$ and are primarily interested in the case where $H$ is a cyclic group of prime power order. Of course, $S G$ (id) is the classical space $S G$ studied by many people. In the classical case the $J$ homomorphism $J$ : $S O \rightarrow S G$ played a pivotal role at the prime 2. Since a linear orthogonal map commutes with the antipodal action $x \rightarrow-x$ on the unit sphere the $J$ homomorphism lifts to a map

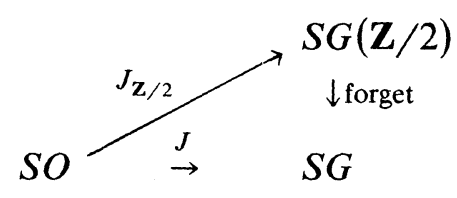

which is compatible with the forgetful map. We shall see that $J_{\mathbf{Z} / 2}$ can not lift to a $\mathbf{Z} / 4$ equivariant $J$ homomorphism for homological grounds in the next section.

REMARK. In this paper we restrict our attention to finite groups whereas Becker and Schultz have defined $G(H)$ for all compact Lie groups $H$. For $H=S^{1}$ there is a complex $J$ homomorphism $J_{S^{1}}: U \rightarrow$ $S G\left(S^{1}\right)$ compatible with the following diagrams:

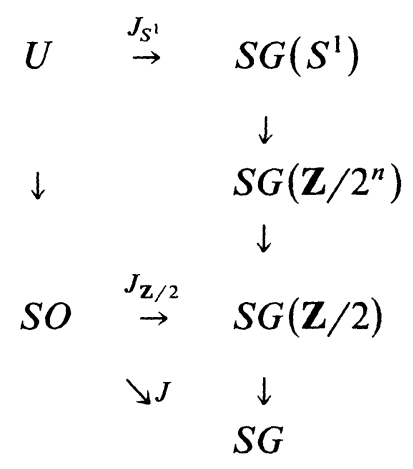


and, for $p$ odd

$$
\begin{array}{lcc}
U & \stackrel{J_{S^{1}}}{\rightarrow} & S G\left(S^{1}\right) \\
& & \downarrow \\
\downarrow & & S G\left(\mathbf{Z} / p^{n}\right) \\
& & \downarrow \\
S O & \stackrel{J}{\rightarrow} & S G
\end{array}
$$

See [13] for an analysis of $J_{S^{1}}$ and its compatibility with 1.1 .8 and 1.1.9.

1.2. The next three sections state the main results of this paper. We postpone all proofs of theorems in these sections to Chapter V. We begin with the characteristic classes for spherical fibrations with fibre-preserving free $\mathbf{Z} / p^{n}$ actions. The reader familiar with the classical results of Milgram [18], May [6], Tsuchiya [33], Madsen [11], and Brumfiel, Madsen, and Milgram [5], will note the extreme similarity with our equivariant results. We assume the reader is familiar with the definitions, structure, and properties of the Dyer-Lashof algebra $R$ (for details, see [6]). To fix our notation, we recall:

(a) $\tilde{H}_{*}(B \mathbf{Z} / 2, \mathbf{Z} / 2) \cong P\left[e_{r} \mid r>0\right]$;

(b) $\tilde{H}_{*}\left(B \mathbf{Z} / 2^{n}, \mathbf{Z} / 2\right) \cong E\left[e_{2 r+1} \mid r \geq 0\right] \otimes P\left[e_{2 r} \mid r>0\right]$ for $n>1$;

(c) $\tilde{H}_{*}\left(B \mathbf{Z} / p^{n}, \mathbf{Z} / p\right) \cong E\left[e_{2 r+1} \mid r \geq 0\right] \otimes P\left[e_{2 r} \mid r>0\right]$ for $p$ odd and all $n>0$; and

TheOREM 1.2.1 [7]. As a Hopf algebra under the loop product

$$
H_{*}\left(Q_{0}\left(B \mathbf{Z} / p^{n^{+}}\right), \mathbf{Z} / p\right) \cong \Lambda\left(x_{I, \alpha}\right)
$$

where $\Lambda\left(x_{I, \alpha}\right)$ is the free commutative algebra on $X_{I, \alpha}=Q^{I}\left(e_{\alpha}\right) *\left(\chi\left(e_{0}\right)\right)^{p^{(I)}}$ where $Q^{I}\left(e_{\alpha}\right)$ is admissible of positive excess.

Here $*$ is the loop sum and $\chi: Q\left(B \mathbf{Z} / p^{n^{+}}\right) \rightarrow Q\left(B \mathbf{Z} / p^{n^{+}}\right)$is the canonical anti-automorphism.

Recall, 1.1.5, that Becker and Schultz showed $Q\left(B \mathbf{Z} / p^{n^{+}}\right)$and $G\left(\mathbf{Z} / p^{n}\right)$ are homotopy equivalent. In Chapter III we prove that $S G\left(\mathbf{Z} / p^{n}\right)$ may be identified as an infinite loop space under composition product with the factor [1] $\times Q_{0}\left(B \mathbf{Z} / p^{n^{+}}\right) \hookrightarrow Q_{1}\left(S^{0}\right) \times Q_{0}\left(B \mathbf{Z} / p^{n^{+}}\right)$in the Segal model for $S F\left(\mathbf{Z} / p^{n}\right)$.

DEFINITION 1.2.2. $\bar{X}_{I, \alpha}=[1] * X_{I, \alpha}$. 
Recall

THEOREM 1.2.3. As a Hopf algebra under the composition product $\tilde{H}_{*}(S O, \mathbf{Z} / 2) \cong E\left[e_{r} \mid r>0\right]$ where

(a) $\operatorname{dim} e_{r}=r$;

(b) $\psi\left(e_{r}\right)=\sum_{i+j=r} e_{i} \otimes e_{j}$ ( $\psi$ is the coalgebra map).

Recall the $\mathbf{Z} / 2$ equivariant $J$ homomorphism, $J_{\mathbf{Z} / 2}, 1.1 .7$, maps $S O$ into $S G(\mathbf{Z} / 2)$.

THEOREM 1.2.4.

(a) $J_{\mathbf{Z} / 2^{*}}: H_{*}(S O, \mathbf{Z} / 2) \rightarrow H_{*}(S G(\mathbf{Z} / 2), \mathbf{Z} / 2)$ is an injection of Hopf algebras.

(b) As a Hopf algebra under the composition product

$$
\begin{aligned}
& H_{*}(S G(\mathbf{Z} / 2), \mathbf{Z} / 2) \\
& \quad \cong H_{*}(S O, \mathbf{Z} / 2) \otimes P\left[J_{\mathbf{Z} / 2}\left(e_{r}\right) * J_{\mathbf{Z} / 2}\left(e_{r}\right) *[-1], \bar{X}_{I, \alpha}\right]
\end{aligned}
$$

where $(I, \alpha)$ is an admissible sequence of positive length and positive excess. The co-algebra structure on the polynomial subalgebra is induced by the Dyer-Lashof algebra.

It is possible to explicitly determine $J_{\mathbf{Z} / 2}\left(e_{r}\right)$ in $H_{*}(\operatorname{SG}(\mathbf{Z} / 2), \mathbf{Z} / 2)$.

THEOREM 1.2.5 [13]. $J_{\mathbf{Z} / 2}\left(e_{r}\right)=\left(e_{r} \circ\left(e_{0} *[-1]\right)\right) *\left([1] * \chi\left(e_{0}\right)\right)=$ $\sum_{i+j=r} \chi\left(e_{i}\right) * Q^{j}\left(e_{0}\right) *[1] * \chi\left(e_{0}\right)$.

For later computations it is convenient to change to a basis of $H_{*}(S G(\mathbf{Z} / 2), \mathbf{Z} / 2)$ that is more natural with respect to the $\mathbf{Z} / 2 \mathrm{~J}$-homomorphism. More precisely, letting

$$
\overline{\bar{X}}_{I, \alpha}=Q^{I}\left(J_{\mathbf{z} / 2}\left(e_{\alpha}\right)\right) *\left(\chi\left(e_{0}\right)\right)^{2^{(I)}} *[1]
$$

we have

THEOREM 1.2.6. As a Hopf algebra under the composition product

$$
\begin{aligned}
& H_{*}(S G(\mathbf{Z} / 2), \mathbf{Z} / 2) \\
& \quad \cong H_{*}(S O, \mathbf{Z} / 2) \otimes P\left[J_{\mathbf{Z} / 2}\left(e_{r}\right) * J_{\mathbf{Z} / 2}\left(e_{r}\right) *[-1], \overline{\bar{X}}_{I, \alpha}\right]
\end{aligned}
$$

where $(I, \alpha)$ is an admissible sequence of positive length and positive excess.

We now consider $S G\left(\mathbf{Z} / 2^{n}\right)$ for $n>1$. 
Consider the composition

$$
U \stackrel{J_{S^{1}}}{\rightarrow} S G\left(S^{1}\right) \stackrel{t_{n}}{\rightarrow} S G\left(\mathbf{Z} / 2^{n}\right)
$$

By results of [13], if $\bar{a}_{r} \in H_{2 r+1}(U, \mathbf{Z} / 2)$ is the standard generator, then

$$
\begin{aligned}
\left(t_{n} \circ J_{S^{1}}\right)_{*} \bar{a}_{r}= & \sum_{s} e_{2 s+1} * \chi e_{2(r-s)} *[1] \\
& +\sum_{t} Q^{2 t+1}\left(e_{2 t}\right) *\left(\chi\left(e_{r-2 t}\right)\right)^{* 2} *[1] .
\end{aligned}
$$

DEFINITION 1.2.7. For $n>1$ let

$$
J_{n}\left(e_{2 r+1}\right)=\left(t_{n} \circ J_{S^{1}}\right)_{*} \bar{a}_{r}
$$

and

$$
\bar{e}_{2 r}=e_{2 r} *[1] * \chi\left(e_{0}\right) .
$$

The following theorem shows there is no $\mathbf{Z} / 2^{n}$ equivariant $J$ homomorphism for $n>1$.

THEOREM 1.2.9. As a Hopf algebra under the composition product

$$
\begin{aligned}
H_{*}\left(S G\left(\mathbf{Z} / 2^{n}\right), \mathbf{Z} / 2\right) \cong & H_{*}(U, \mathbf{Z} / 2) \otimes P\left(\bar{e}_{2 r}\right) \\
& \otimes P\left[\left(J_{n}\left(e_{2 r+1}\right) * J_{n}\left(e_{2 r+1}\right) *[-1]\right), \bar{X}_{I, \alpha}\right] .
\end{aligned}
$$

Here $n>1$ and $(I, \alpha)$ is an admissible sequence of positive length and positive excess.

Again, the coalgebra structure is induced from the Dyer-Lashof algebra.

THEOREM 1.2.10. Let $p$ be an odd prime. As a Hopf algebra under the composition product

$$
H_{*}\left(S G\left(\mathbf{Z} / p^{n}\right), \mathbf{Z} / p\right) \cong H_{*}\left(Q_{0}\left(B \mathbf{Z} / p^{n^{+}}\right), \mathbf{Z} / p\right) .
$$

That is, the composition and loop Hopf algebra structures are abstractly isomorphic.

As in the classical case there does not exist a map inducing the isomorphism in 1.2.10.

We now turn to the classifying space level. First let $p=2$. 
Definition 1.2.11. $B\left(\mathbf{Z} / 2^{n}\right)=P\left[s\left(\bar{X}_{L, \alpha}\right)\right]$ is the primitively generated Hopf algebra on $s\left(\bar{X}_{I, \alpha}\right)$, where degree $s\left(\bar{X}_{I, \alpha}\right)=$ degree $\bar{X}_{I, \alpha}+1,(I, \alpha)$ is an admissible sequence with $e(I, \alpha) \geq 2$ if $l(I, \alpha) \geq 2$ and

(a) for $n=1, e(I, \alpha) \geq 1$ if $l(I, \alpha)=1$;

(b) for $n>1, e(I, 2 \alpha+1) \geq 1$ if $l(I, 2 \alpha+1)=1, e(I, 2 \alpha) \geq 2$ if $l(I, 2 \alpha)=1$

(c) for $n>1$ the classes $\bar{X}_{\phi, 2 \alpha}=e_{2 \alpha} * \chi\left(e_{0}\right) * 1$ are in $B\left(\mathbf{Z} / 2^{n}\right)$.

THEOREM 1.2.12. As a Hopf algebra

$$
\begin{aligned}
H_{*}(B S G(\mathbf{Z} / 2), \mathbf{Z} / 2) \cong & H_{*}(B S O, \mathbf{Z} / 2) \\
& \otimes E\left[s\left(J_{\mathbf{Z} / 2}\left(e_{r}\right) * J_{\mathbf{Z} / 2}\left(e_{r}\right) *[-1]\right)\right] \otimes B(\mathbf{Z} / 2) .
\end{aligned}
$$

THEOREM 1.2.13. Let $n>1$, then as a Hopf algebra

$$
\begin{aligned}
H_{*}( & \left.B S G\left(\mathbf{Z} / 2^{n}\right), \mathbf{Z} / 2\right) \\
\cong & H_{*}(B U, \mathbf{Z} / 2) \otimes E\left[s\left(J_{\mathbf{Z} / 2}\left(e_{2 r+1}\right) * J_{\mathbf{Z} / 2}\left(e_{2 r+1}\right) *[-1]\right)\right] \\
& \otimes B\left(\mathbf{Z} / 2^{n}\right) .
\end{aligned}
$$

Now let $p$ be odd.

THEOREM 1.2.14. For $p$ odd and all $n \geq 1$

$$
H_{*}\left(B S G\left(\mathbf{Z} / p^{n}\right), \mathbf{Z} / p\right) \cong H_{*}(B S O, \mathbf{Z} / p) \otimes S
$$

as Hopf algebras where $S$ is a primitively generated free commutative algebra.

1.3. In this section we give the determinations of the forgetful maps $t: S G\left(H_{2}\right) \rightarrow S G\left(H_{1}\right)$ for the spaces in the previous section. Segal and Becker and Schultz have identified the forgetful map up to homotopy with the transfer map $t: Q\left(B H_{2}^{+}\right) \rightarrow Q\left(B H_{1}^{+}\right)$which is an infinite loop map with respect to the loop sum structure. As the forgetful map is naturally an infinite loop space with respect to the composition product structure, it behaves well in homology with respect to both products and their associated Dyer-Lashof operations. Thus it suffices to determine $t: S G\left(\mathrm{H}_{2}\right) \rightarrow$ $S G\left(H_{1}\right)$ on the cells of $\mathrm{BH}_{2}^{+}$.

THEOREM 1.3.1. The transfers

$$
t\left(2^{n}, 2^{n-1}\right): H_{*}\left(S G\left(\mathbf{Z} / 2^{n}\right), \mathbf{Z} / 2\right) \rightarrow H_{*}\left(S G\left(\mathbf{Z} / 2^{n-1}\right), \mathbf{Z} / 2\right)
$$

are completely determined by the following formulae. 
(a) $t(2,1) e_{i}=Q^{i}[1]$

(b) $t(4,2) e_{2 i}=Q^{i} e_{i}, t(4,2) e_{2 i+1}=\sum_{j=0}^{i} e_{2 j+1} * e_{2(i-j)}$;

(c) for $n>2$,

$$
\begin{aligned}
t\left(2^{n}, 2^{n-1}\right) e_{4 i} & =Q^{2 i} e_{2 i}, \\
t\left(2^{n}, 2^{n-1}\right) e_{4 i+1} & =Q^{2 i+1} e_{2 i}+\sum_{j=0}^{2 i} e_{2 j} * e_{4 i-2 j+1}, \\
t\left(2^{n}, 2^{n-1}\right) e_{4 i+2} & =0, \\
t\left(2^{n}, 2^{n-1}\right) e_{4 i+3} & =\sum_{j=0}^{2 i} e_{2 j} * e_{4 i-2 j+3} .
\end{aligned}
$$

COROLlaRY 1.3.2. The transfers

$$
t\left(2^{n}, 2^{n-1}\right): H_{*}\left(S G\left(\mathbf{Z} / 2^{n}\right), \mathbf{Z} / 2\right) \rightarrow H_{*}\left(S G\left(\mathbf{Z} / 2^{n-1}\right), \mathbf{Z} / 2\right)
$$

are not surjective for $n>1$.

Corollary 1.3.2 gives another proof that the classical $J$ homomorphism may not be lifted to $S G\left(\mathbf{Z} / 2^{n}\right)$ for $n>1$.

Theorem 1.3.1 is sufficient to explicitly calculate all transfers induced by the inclusions $\mathbf{Z} / 2^{m}-\mathbf{Z} / 2^{n}$ for all $m<n$. We state one explicit result.

THEOREM 1.3.3. The transfers

$$
t\left(2^{n}, 1\right): H_{*}\left(S G\left(\mathbf{Z} / 2^{n}\right), \mathbf{Z} / 2\right) \rightarrow H_{*}(S G, \mathbf{Z} / 2)
$$

are completely determined by the following formulae.

(a) $t(2,1) e_{i}=Q^{i}(1)$

(b) $t(4,1) e_{2 i}=Q^{i} Q^{i}(1)=\left(Q^{i}(1)\right)^{* 2}$,

$$
t(4,1) e_{2 i+1}=Q^{i+1} Q^{i}(1)+\sum_{j=0}^{i} Q^{2 j+1}(1) * Q^{2(i-j)}(1) ;
$$

(c) for $n>2$,

$$
\begin{aligned}
t\left(2^{n}, 1\right)\left(e_{2^{n-1} \cdot M}\right)= & \left(Q^{M}(1)\right)^{* 2^{n-1}}, \\
t\left(2^{n}, 1\right)\left(e_{2 N}\right)= & 0 \quad \text { if } 2^{n-2}+N ; \\
t\left(2^{n}, 1\right)\left(e_{2 d+1}\right)= & \sum\left(Q^{j_{1}}(1)\right) * 2^{n-1} *\left(Q^{j_{2}}(1)\right)^{2^{n-3}} * \cdots *\left(Q^{j_{l}}(1)\right) * 2 \\
& *\left[Q^{2 k+1}(1) * Q^{2 t}(1)+Q^{k+t+1} Q^{k+t}(1)\right]
\end{aligned}
$$


where the sum runs over all $j_{1}, \ldots, j_{l}, k, t$ so that

$$
\begin{gathered}
\sum_{r=1}^{n-2} 2^{n-2-r} \cdot j_{r} \leq 2 d ; \\
k, t \geq 0 ; \quad t=d-\sum_{r=1}^{n-2} 2^{n-3-r} j_{r}-k .
\end{gathered}
$$

One obtains analogous results for odd primes.

THEOREM 1.3.4. The transfers

$$
t\left(p^{n}, p^{n-1}\right): H_{*}\left(S G\left(\mathbf{Z} / p^{n}\right), \mathbf{Z} / p\right) \rightarrow H_{*}\left(S G\left(\mathbf{Z} / p^{n-1}\right), \mathbf{Z} / p\right)
$$

for $p$ an odd prime are completely determined by the following formulae.

(a) $t(p, 1)\left(e_{2 s(p-1)}\right)=(-1)^{s} Q^{s}(1)$

$$
\begin{aligned}
& t(p, 1)\left(e_{2 s(p-1)-1}\right)=(-1)^{s} \beta Q^{s}(1), \\
& t(p, 1)\left(e_{i}\right)=0 \text { otherwise; }
\end{aligned}
$$

(b) for $n>1$,

$$
\begin{gathered}
t\left(p^{n}, p^{n-1}\right)\left(e_{p(2 m)}\right)=Q^{2 m}\left(e_{2 m}\right)=\left(e_{2 m}\right) * p \\
t\left(p^{n}, p^{n-1}\right)\left(e_{p(2 m+2 d+1)}\right) \\
=\sum \alpha\left(2 m_{1}+2 d+1, m_{2}, \ldots, m_{p}\right) e_{2 m_{1}+2 d+1} * e_{2 m_{2}} * \cdots * e_{2 m_{p}},
\end{gathered}
$$

where

(i) $0 \leq d<(p-1) / 2$.

(ii) The sum runs over all nonnegative $m_{1}, \ldots, m_{p}$ such that $\sum_{i=1}^{p} m_{i}=$ $m$.

(iii) The coefficient $\alpha\left(j_{1}, \ldots, j_{p}\right)$ is the number, taken mod $p$, of permutations $\sigma$ of $1,2, \ldots, p$ such that $\left(j_{1}, j_{2}, \ldots, j_{p}\right)=\left(j_{\sigma(1)}, j_{\sigma(2)}, \ldots, j_{\sigma(p)}\right)$. And finally

$$
t\left(p^{n}, p^{n-1}\right)\left(e_{\imath}\right)=0 \quad \text { otherwise. }
$$

THEOREM 1.3.5. The transfers

$$
t\left(p^{n}, 1\right): H_{*}\left(S G\left(\mathbf{Z} / p^{n}\right), \mathbf{Z} / p\right) \rightarrow H_{*}(S G, \mathbf{Z} / p)
$$

for $p$ an odd prime are completely determined by the following formulae.

(a) $t(p, 1) e_{2 s(p-1)}=(-1)^{s} Q^{s}(1)$,

$$
t(p, 1) e_{2 s(p-1)-1}=(-1)^{s} \beta Q^{s}(1)
$$$$
t(p, 1) e_{\imath}=0 \text { otherwise; }
$$ 
(b) $t\left(p^{2}, 1\right)\left(e_{2 s(p-1) p}\right)=(-1)^{s} Q^{2 s} Q^{s}(1)=(-1)^{s}\left(Q^{s}(1)\right)^{* p}$, $t\left(p^{2}, 1\right)\left(e_{2 M}\right)=0$ if $p(p-1) \nmid M$, $t\left(p^{2}, 1\right)\left(e_{2 N-1}\right)=0$ if $p-1 \nmid N$,

$$
\begin{aligned}
& t\left(p^{2}, 1\right)\left(e_{2 d(p-1)-1}\right) \\
& \quad=(-1)^{d} \sum \alpha\left(\cdot, s_{2}, \ldots, s_{p}\right) \beta Q^{s_{1}}(1) * Q^{s_{2}}(1) * \cdots * Q^{s_{p}}(1)
\end{aligned}
$$

where the sum runs over all nonnegative $s_{1}, \ldots, s_{p}$ such that $\sum_{i=1}^{p} s_{i}=d$ and $\alpha\left(\cdot, s_{2}, \ldots, s_{p}\right)$ is defined as in 1.3.4;

(c) for $n>2$,

$$
\begin{gathered}
t\left(p^{n}, 1\right) e_{2 s(p-1) p^{n-1}}=(-1)^{s}\left(Q^{s}(1)\right)^{* p^{n-1}}, \\
t\left(p^{n}, 1\right) e_{2 M}=0 \quad \text { if }(p-1) p^{n-1} \nmid M, \\
t\left(p^{n}, 1\right) e_{2 N-1}=0 \quad \text { if }(p-1) \nmid N, \\
t\left(p^{n}, 1\right) e_{2 d(p-1)-1} \\
=(-1)^{d} \sum(\operatorname{coeff}) \beta Q^{s_{n-1,1}}(1) * Q^{s_{n-1,2}}(1) * \cdots * Q^{s_{n-1, p}}(1) \\
*\left(Q^{s_{n-2,2}}(1) * \cdots * Q^{s_{n-2, p}}(1)\right) * p \\
* \cdots *\left(Q^{s_{1,2}}(1) * \cdots * Q^{s_{1, p}}(1)\right)^{n p^{n-2}},
\end{gathered}
$$

where the sum runs over $s_{n-1,1}$ and all $s_{a, b}, 1 \leq a \leq n-1,2 \leq b \leq p$ such that $\sum_{c=1}^{n-1}\left[p^{c-1} \sum_{i=2}^{p} s_{n-c, i}\right]=d-s_{n-1,1}$ and the coefficient, coeff, is the product $\prod_{c=1}^{n-1} \alpha\left(\cdot, s_{n-c, 2}, \ldots, s_{n-c, p}\right)$.

1.4. In this section we examine the homological implications of the geometric fact that a real line bundle may be tensored with a $\mathbf{Z} / 2$ equivariant spherical fibration. Thus we let $H=\mathbf{Z} / 2$ and concentrate our attention on $G(\mathbf{Z} / 2)$ and $B G(\mathbf{Z} / 2)$. In fact the original motivation for this paper was to obtain Theorem 1.4.8 which is used in [14] to study mapping tori and projective bundle constructions in unoriented $P L$ cobordism.

We now consider $G(\mathbf{Z} / 2)_{ \pm 1}$ the \pm 1 component of $G(\mathbf{Z} / 2)$ consisting of stable $\mathbf{Z} / 2$ equivariant $V_{\mathbf{Z} / 2}$ spherical homotopy equivalences. As mentioned in the introduction, [1], [15], and [34] imply $B G(\mathbf{Z} / 2)_{ \pm 1}$ classifies stable virtue spherical fibrations with free fibre-preserving involutions ( $B S G(\mathbf{Z} / 2)$ classifies the oriented theory).

Now given a real line bundle $L$ over $M$ and a spherical fibration over $M$ with free involution, $(\xi, t)$, one naturally obtains a new spherical fibration $L \otimes_{t} \xi$ over $M$ which also has an involution induced by $t$. 
Definition 1.4.1. $L \otimes_{t} \xi$ is the space $\tilde{M} \times_{\mathrm{z} / 2} \xi$ where $\tilde{M}$ is the unique double cover of $M$ associated to $L$. Denote by $\bar{t}$ the involution $t$ induces on $L \otimes_{t} \xi$.

Hence by considering virtual bundles $L \otimes_{t}(\xi-N)=L \otimes_{t} \xi-L \otimes N$ (here $N=\operatorname{dim} \xi$ ), we obtain a map

$$
\psi_{G}: \mathbf{R} P^{\infty} \times B G(\mathbf{Z} / 2)_{ \pm 1} \rightarrow B G(\mathbf{Z} / 2)_{ \pm 1} .
$$

Of course if $\xi$ is a vector bundle with the standard involution, we recover the standard tensor product so $\psi_{G}$ covers the standard smooth map; that is, the following diagram commutes.

$$
\begin{array}{lll}
\psi_{G}: \mathbf{R} P^{\infty} \times B G(\mathbf{Z} / 2)_{ \pm 1} & \rightarrow & B G(\mathbf{Z} / 2)_{ \pm 1} \\
\uparrow \mathrm{id} \times B J_{\mathbf{Z} / 2} & & \uparrow B J_{\mathbf{Z} / 2} \\
\psi_{0}: \mathbf{R} P^{\infty} \times B O & \rightarrow & B O
\end{array}
$$

For $\xi$ of virtual dimension zero, $\psi_{G}$ and $\psi_{0}$ map $\mathbf{R} P^{\infty} \times$ base point to the base point. Thus we may loop the map in 1.4.3 to obtain

$$
\begin{array}{lll}
\mathbf{R} P^{\infty} \times Q\left(\mathbf{R} P^{\infty+}\right) & \rightarrow & Q\left(\mathbf{R} P^{\infty+}\right) \\
\uparrow & & \jmath \\
\Omega \psi_{G}: \mathbf{R} P^{\infty} \times \Omega B G(\mathbf{Z} / 2)_{ \pm 1} & \rightarrow & \Omega B G(\mathbf{Z} / 2)_{ \pm 1} \\
\uparrow \text { id } \times J_{\mathbf{Z} / 2} & & \uparrow J_{\mathbf{Z} / 2} \\
\Omega \psi_{0}: \mathbf{R} P^{\infty} \times \Omega B O & \rightarrow & \Omega B O \\
\uparrow & & \uparrow \\
\mathbf{R} P^{\infty} \times S O & \rightarrow & S O
\end{array}
$$

Let us fix the following notation.

(1) $\tilde{H}_{*}(S O, \mathbf{Z} / 2) \cong E\left[x_{r} \mid r>0\right]$ where $x_{r}$ is represented by the image of $\mathbf{R} P^{r} \hookrightarrow S O$ given by sending a line $l$ in $\mathbf{R}^{n}$ to reflection perpendicular to $l$ times a fixed rotation.

(2) $\tilde{H}_{*}(B O, \mathbf{Z} / 2) \cong P\left[z_{s} \mid s>0\right]$ where $z_{s}$ is represented by the image of $\mathbf{R} P^{s} \hookrightarrow \mathbf{R} P^{\infty} \hookrightarrow B O$ where the inclusion $\mathbf{R} P^{\infty} \hookrightarrow B O$ classifies the virtual non-trivial line bundle over $\mathbf{R} P^{\infty}$.

(3) Let $y_{s} \in \tilde{H}_{*}(B O, \mathbf{Z} / 2)$ be the class represented by the image of $S^{1} \wedge \mathbf{R} P_{+}^{s} \rightarrow S^{1} \wedge O_{+} \rightarrow B O$ where the first inclusion is given by $S^{1} \wedge x_{s}$ and the second map is the adjoint to $0 \sim \Omega B O$.

(4) Let $e_{t}$ represent the generator of $H_{t}\left(\mathbf{R} P^{\infty}, \mathbf{Z} / 2\right)$. 
Of course, $\psi_{0}$ is approximated by the maps $\mathbf{R} P^{\infty} \times B O(N) \rightarrow B O$ which classify $L \otimes\left(E_{N}-\bar{N}\right)$. The following theorems are proved in Chapter V.

THEOREM 1.4.5.

(a) $\psi_{0_{*}}\left(e_{t} \otimes z_{s}\right)=\Sigma_{s=p+q}\left(\begin{array}{c}t+p \\ p\end{array}\right) z_{t+p} \cdot \chi\left(z_{q}\right)$;

(b) $\psi_{0_{*}}\left(e_{t} \otimes y_{s}\right)=\left(\begin{array}{c}t+s \\ t\end{array}\right) y_{s+t}$;

(c) $y_{s}=\sum_{w=\left(i_{1}, \ldots, i_{r}\right)} N(w) z_{i_{1}} \cdot z_{i_{2}} \cdots \cdot z_{i_{r}}$ where $N(w)$ is the coefficient in the expansion $S_{w}(\sigma)=N(w) w_{\left(\Sigma_{\alpha=1}^{r} i_{\alpha}\right)}+$ decomposables in $H_{*}(B O)$;

(d) $\left(\Omega \psi_{0}\right)_{*}\left(e_{t}, x_{r}\right)=\left(\begin{array}{c}t+r \\ r\end{array}\right) x_{t+r}$.

THEOREM 1.4.6. The map $\Omega \psi_{G}$ is a homology pairing for both loop sum and composition product structures. More precisely, we have the following formulae.

(a) $\Omega \psi_{G_{*}}\left(e_{t}, x * y\right)=\Sigma_{a+b=t} \Omega \psi_{G_{*}}\left(e_{a}, x\right) * \Omega \psi_{G_{*}}\left(e_{b}, y\right)$;

(b) $\Omega \psi_{G_{*}}\left(e_{t}, Q^{r}(x)\right)=\sum_{a \geq 0} Q^{r+a}\left(\Omega \psi_{G_{*}}\left(\left(S q_{*}^{a}\right) e_{t}, x\right)\right)$;

(c) $\Omega \psi_{G_{*}}\left(e_{t}, x \cdot y\right)=\Sigma_{a+b=t} \Omega \psi_{G_{*}}\left(e_{a}, x\right) \cdot \Omega \psi_{G_{*}}\left(e_{b}, y\right)$;

(d) $\Omega \psi_{G_{*}}\left(e_{t}, \hat{Q}^{r}(x)\right)=\sum_{a \geq 0} \hat{Q}^{r+a}\left(\Omega \psi_{G_{*}}\left(\left(S q_{*}^{a}\right) e_{t}, x\right)\right)$.

Theorems 1.4.5, 1.4.6 and the fact that $J_{\mathbf{Z} / 2}$ is an infinite loop map determine $\Omega \psi_{G_{*}}$. In Chapter $\mathrm{V}$ we recall a weight valuation first used by May [6], and Tsuchiya [33], on $H_{*}\left(Q\left(S^{0}\right), \mathbf{Z} / p\right)$ and extend it to give a filtration on $H_{*}\left(Q\left(B \mathbf{Z} / p^{\mathbf{n}^{+}}\right), \mathbf{Z} / p\right)$. We then have

THEOREM 1.4.7. Modulo higher weight

$$
\begin{aligned}
\Omega \psi_{G_{*}} & \left(e_{j}, Q^{I}\left(J_{\mathbf{Z} / 2}\left(e_{r}\right)\right)\right) *\left([1] *\left(\chi\left(e_{0}\right)^{2^{2(t)}}\right)\right) \\
= & \sum_{T} c(T) Q^{I+T}\left(J_{\mathbf{Z} / 2}\left(e_{r+j-s}\right)\right) *\left([1] *\left(\chi\left(e_{0}\right)\right)^{2^{((r)}}\right)
\end{aligned}
$$

where

(1) the sum runs over all $T=\left(t_{1}, \ldots, t_{k}\right)$ with each $t_{l} \geq 0$.

(2) $I+T=\left(i_{1}+t_{1}, i_{2}+t_{2}, \ldots, i_{k}+t_{k}\right)$.

(3) $s=\sum_{l=1}^{k} t_{l}$.

(4) $c(T)$ is the following product of binomial coefficients:

$\left(j-2 t_{1}, t_{1}\right)\left(j-t_{1}-2 t_{2}, t_{2}\right) \cdots\left(j-t_{1}-t_{2}-\cdots-2 t_{k}, t_{k}\right)(r, j-s)$.

Here we use the notation $(a, b)=\left(\begin{array}{c}a+b \\ a\end{array}\right)$.

REMARK. 1.4.7 may be restated by replacing the statement "modulo higher weight" by the statement "modulo loop decomposables." 
Recalling that $\sigma_{*}$ is the homology suspension, we conclude this section with

THEOREM 1.4.8. Modulo higher weight

$$
\begin{aligned}
\psi_{G_{*}}\left(e_{j}, \sigma_{*}\left(Q^{I}\left(J_{\mathbf{Z} / 2}\left(e_{r}\right)\right) *\left([1] *\left(\chi\left(e_{0}\right)\right)^{2^{(I I)}}\right)\right)\right) \\
\quad=\sum_{T} c(T) \sigma_{*}\left(Q^{I+T}\left(J_{\mathbf{z} / 2}\left(e_{r+j-s}\right)\right) *\left([1] *\left(\chi\left(e_{0}\right)\right)^{2^{(I I)}}\right)\right)
\end{aligned}
$$

where $T, I+T, s$, and $c(T)$ are as defined in 1.4.7.

\section{CHAPTER II}

In this chapter we use the $E_{\infty}$ ring space machinery of May and his school [16], [17], and [9] to construct a good $E_{\infty}$ ring model for $F(H)$. The preliminary version of this chapter was revised after receiving a helpful letter from J. P. May whom we would like to thank for his advice and comments.

We begin by recalling a specific $E_{\infty}$ operad pair. The additive operad $\mathscr{K}(\bar{j})$ was recently discovered by Richard Steiner [30].

Let $\bar{j}$ be the finite right $H$-set $\{1,2, \ldots, j\}$ and let $W$ be any $H$ invariant finite dimensional subspace of $(V \oplus \mathbf{R})_{H}=\cup_{m, n}\left(m V \oplus \mathbf{R}^{n}\right)$. Give $\bar{j} \times W$ the diagonal $H$ action.

Definition 2.1.1. [30] $\mathcal{K}(\bar{j}, W)$ is the space of homotopies $f_{t}: \bar{j} \times W$ $\rightarrow W, 0 \leq t \leq 1$, such that

(a) $\left.f_{t}\right|_{y \times W}: y \times W \rightarrow W$ is a homeomorphism of $y \times W$ onto its image, which is open, for each $y \in \bar{j}$ and for all $0 \leq t \leq 1$;

(b) $\left.f_{0}\right|_{y \times W}: y \times W \rightarrow W$ maps $(y, \vec{x})$ to $\vec{x}$ for all $x \in W$;

(c) $f_{1}$ is an embedding of $\bar{j} \times W$ into $W$.

Let $W \subset Z$ be $H$ invariant finite dimensional subspaces of $(V \oplus \mathbf{R})_{H}$. We define a map

$$
\mathscr{K}(\bar{j}, W, Z): \mathscr{K}(j, W) \rightarrow \mathscr{K}(j, Z)
$$

by the composition

$$
y \times Z=y \times\left(W \oplus\left(W^{\perp} \cap Z\right)\right) \stackrel{f_{t} \oplus \text { id }}{\rightarrow} W \oplus\left(W^{\perp} \cap Z\right)=Z
$$

for all $y \in Y$. This allows us to pass to the limit of the $\mathscr{K}(\bar{j}, W)$ 's. 
Definition 2.1.3 [30]. $\mathscr{K}(\bar{j})=\lim _{\rightarrow} \mathscr{K}(\bar{j}, W)$ where $W$ ranges over all $H$ invariant finite dimensional subspaces of $(V \oplus \mathbf{R})_{H}$.

As $\bar{j} \times W$ has the diagonal $H$ action $H$ acts on $\mathscr{K}(\bar{j}, W)$ and thus on $\mathscr{K}(\bar{j})$ via conjugation.

THEOREM 2.1.4 [30]. $\mathscr{K}(\bar{j})$ is an $E_{\infty}$ operad.

See [30] for full details. We merely note that the $E_{\infty}$ operad structure is given by letting $\Sigma_{j}$ permute the $f_{t}^{i}$,s and by the mapping $\gamma_{\mathcal{F}}: \mathcal{K}(j) \times$ $\mathcal{K}\left(i_{1}\right) \times \cdots \times \mathcal{K}\left(i_{j}\right) \rightarrow \mathcal{K}\left(i_{1}+\cdots+i_{j}\right)$ given by

$$
\begin{aligned}
& \gamma_{\mathcal{K}}\left(\left(f_{t}{ }^{1}, \ldots, f_{t}^{j}\right) ;\left(g_{t}^{11}, \ldots, g^{1 i_{1}}\right), \ldots,\left(g_{t}^{j 1}, \ldots, g_{t}^{j i_{j}}\right)\right) \\
& \quad=\left(\left(f_{t}{ }^{1} \cdot g_{t}^{11}\right), \ldots,\left(f_{t}{ }^{1} \cdot g_{t}^{1 i_{1}}\right), \ldots,\left(f_{t}^{j} \cdot g_{t}^{j 1}\right), \ldots,\left(f_{t}^{j} \cdot{ }^{j i_{j}}\right)\right) .
\end{aligned}
$$

The multiplicative operad we consider was first used by Boardman and Vogt [4], and May [16].

TheOREM 2.1.5 [4], [16]. $\mathcal{L}(j)=\operatorname{Iso}\left((V \oplus \mathbf{R})_{H}^{j},(V \oplus \mathbf{R})_{H}\right)$, the space of isometries of $(V \oplus \mathbf{R})_{H}^{j}$ into $(V \oplus \mathbf{R})_{H}$ is an $E_{\infty}$ operad.

Again note that $H$ acts on $\mathcal{L}(j)$ via conjugation.

See [4] or [16] for full details. Again, we merely recall that $\Sigma_{j}$ acts freely on $\mathcal{L}(j)$ by permuting the factors of $(V \oplus \mathbf{R})_{H}^{j}$, and the structure map $\gamma_{\mathfrak{L}}$ that makes $\mathscr{L}(j)$ an $E_{\infty}$ operad $\gamma_{\mathfrak{L}}: \mathcal{L}(j) \times \mathfrak{L}\left(i_{1}\right) \times \cdots \times \mathfrak{L}\left(i_{j}\right) \rightarrow$ $\mathcal{L}\left(i_{1}+\cdots+i_{j}\right)$ is given by $\gamma_{\mathcal{E}}\left(f ; g_{1}, \ldots, g_{j}\right)=f \circ\left(g_{1} \times \cdots \times g_{j}\right)$.

Notice now that $\mathcal{L}$ acts on $\mathcal{K}$ as follows. We define a map

$$
\lambda: \mathscr{L}(j) \times \mathscr{K}\left(i_{1}\right) \times \cdots \times \mathfrak{K}\left(i_{j}\right) \rightarrow \mathscr{K}\left(i_{1} \cdots i_{j}\right)
$$

by

$$
\begin{aligned}
& \lambda\left(f ;\left(\left(g_{t}^{11}, \ldots, g_{t}^{1 i_{1}}\right), \ldots,\left(g_{t}^{j 1}, \ldots, g_{t}^{j i_{j}}\right)\right)\right) \\
& \quad=\left\{f \circ\left(g_{t}^{1 \alpha_{1}} \times g_{t}^{2 \alpha_{2}} \times \cdots \times g_{t}^{j \alpha_{j}}\right) \circ f^{-1}\right\}_{1 \leq \alpha_{r} \leq i_{r} ; 1 \leq r \leq j}
\end{aligned}
$$


where we have lexicographically ordered the $g_{t}^{a b}$ 's. By $f \circ\left(g^{1 \alpha_{1}}\right.$ $\left.\times \cdots \times g^{j \alpha_{j}}\right) \circ f^{-1}$ we mean the composition of maps given by

$$
\begin{aligned}
& (V \oplus \mathbf{R})_{H}=f\left((V \oplus \mathbf{R})_{H}^{j}\right) \oplus\left[f\left((V \oplus \mathbf{R})_{H}^{j}\right)\right]^{\perp} \\
& \stackrel{f^{-1} \oplus \text { id }}{\rightarrow}(V \oplus \mathbf{R})_{H}^{j} \oplus\left[f\left((V \oplus \mathbf{R})_{H}^{j}\right)\right]^{\perp} \\
& \stackrel{\left(g^{1, \alpha_{1}} \times \cdots \times g^{j, \alpha_{j}}\right) \oplus \mathrm{id}}{\rightarrow}(V \oplus \mathbf{R})_{H}^{j} \oplus\left[f\left((V \oplus \mathbf{R} j)_{H}^{j}\right)\right]^{\perp} \\
& \stackrel{f \oplus \mathrm{id}}{\rightarrow} f\left((V \oplus \mathbf{R})_{H}^{j}\right) \oplus\left[f\left((V \oplus \mathbf{R})_{H}^{j}\right)\right]^{\perp}=(V \oplus \mathbf{R})_{H} .
\end{aligned}
$$

This map is well defined as $f$ is an isometry and hence a linear isomorphism onto its image. The resulting map is thus a homeomorphism onto an open set in $(V \oplus \mathbf{R})_{H}$ for each $0 \leq t \leq 1$ and $\alpha_{1}, \ldots, \alpha_{j}$. Furthermore, it is the identity for $t=0$ and all factors have disjoint images for $t=1$. Hence we obtain an element in $\mathscr{K}\left(i_{1} \cdots i_{j}\right)$.

A long but direct check of the conditions in [16] or [17] shows

THEOREM 2.1.7. ( $K, \mathcal{L}, \lambda)$ is an $E_{\infty}$ operad pair.

Proof: [30].

2.2. In this section we summarize results we require from [9] to obtain an $E_{\infty}$ ring version of the Segal configuration map. Let $K$ be the realization of the $\mathcal{K}$ operad acting on the trivial $H$ space in the standard monad construction. That is,

$$
K=\coprod_{j} \mathcal{K}(j) / \Sigma_{j}
$$

where we note $K$ has the obvious $H$ action.

Let $W$ be any $H$ invariant finite dimensional subspace of $(V \oplus \mathbf{R})_{H}$ and consider $F(t W, t W)$, the space of pointed self maps of the one point compactification of $W$. Note that $F(t W, t W)$ has a natural $H$ action induced by conjugation.

We now define the configuration map

$$
\psi_{W}: \mathfrak{K}(\bar{j}, W) \rightarrow F(t W, t W)
$$


as follows: Given $f_{t} \in \mathscr{K}_{H}(\bar{j}, W)$

$$
\psi_{W}\left(f_{t}\right)(\vec{x})= \begin{cases}\infty & \text { if } \vec{x}=\infty, \\ \infty & \text { if } \vec{x} \notin f_{1}(j \times W), \\ \left(\pi \cdot f_{1}^{-1}\right) \vec{x} & \text { if } \vec{x} \in f_{1}(\bar{j} \times W),\end{cases}
$$

where $\pi: \bar{j} \times W \rightarrow W$ is the natural projection. A trivial check shows $\psi_{W}$ is compatible with taking limits as $W$ ranges over all finite dimensional $H$ invariant subspaces of $(V \oplus \mathbf{R})_{H}$ (see Remark 2 following 1.1.2 and 2.1.2) and thus we obtain the configuration map first considered by Segal

$$
\psi: K \rightarrow \underset{W}{\lim } F(t W, t W)=F .
$$

Next we have the following result, due to Hauschild, May, and Waner.

THEOREM 2.2.4 [9]. $\psi$ is an $E_{\infty}$ ring map of $E_{\infty}$ ring spaces where the $E_{\infty}$ ring structure is given by the operad $(\mathfrak{K}, \mathcal{L})$.

Proof. [9].

We further specialize 2.2 .4 by considering the $H$-fixed point sets of 2.2.3 to obtain the configuration map we are interested in

$$
\phi=\psi^{H}: K^{H} \rightarrow F^{H}=F(H) .
$$

COROLlaRY 2.2.6 [9]. $\phi: K^{H} \rightarrow F(H)$ is an $E_{\infty}$ ring map of $E_{\infty}$ ring structure is given by the operad $\left(\mathscr{K}^{H}, \mathfrak{L}^{H}\right)$.

Proof of 2.2.4. We describe how $\mathscr{K}$ and $\mathcal{L}$ act on both $K$ and $F$. The rest of the proof is straightforward and left to the reader.

(a) The action $\mathcal{L}(k) \times K^{k} \rightarrow K$ is induced by the maps

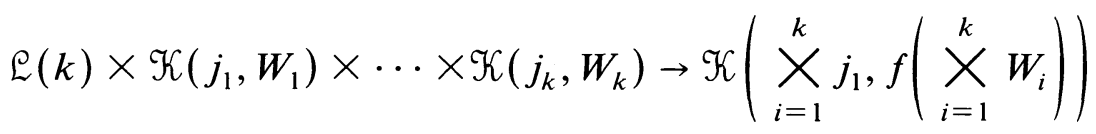

where given $f \in \operatorname{Iso}\left((V \oplus \mathbf{R})_{H}^{k},(V \oplus \mathbf{R})\right)_{H}$ and $g_{t}^{i} \in \mathcal{K}\left(j_{i}, W_{i}\right)$ we obtain a map

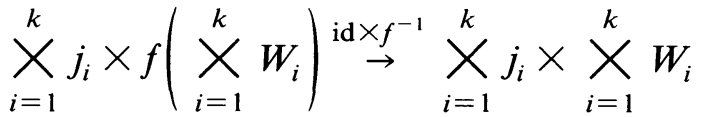

$$
\begin{aligned}
& \stackrel{\text { shuffle }}{\rightarrow} \underset{i=1}{\underset{X}{X}}\left(j_{i} \times W_{i}\right) \stackrel{\stackrel{i=1}{x}_{\rightarrow}^{k} g_{t}^{i}}{\underset{X}{X}} W_{i=1} \stackrel{f}{\rightarrow} f\left(\underset{i=1}{\underset{X}{X} W_{i}}\right)
\end{aligned}
$$


which is easily verified to be an element of $\mathscr{K}\left(\times_{i=1}^{k} j_{i}, f\left(\times_{i=1}^{k} W_{i}\right)\right)$. This is compatible with the $H$ action and with passage to the limit.

(b) The action $\mathcal{L}(k) \times F^{k} \rightarrow F$ is similarly induced as follows. Let $f \in \operatorname{Iso}\left((V \oplus \mathbf{R})_{H},(V \oplus \mathbf{R})_{H}\right)$ and $g_{i} \in F\left(t W_{i}, t W_{i}\right)$ for $1 \leq i \leq k$. Then define the self map $t f\left(\times_{i=1}^{k} W_{i}\right)$ by the composition

$$
\begin{aligned}
& t f\left({\underset{i=1}{X} W_{i}}^{\stackrel{f^{-1}}{\rightarrow} t}\left(\underset{i=1}{\underset{X}{X}} W_{i}\right)=t W_{1} \wedge t W_{2} \wedge \cdots \wedge t W_{k}\right.
\end{aligned}
$$

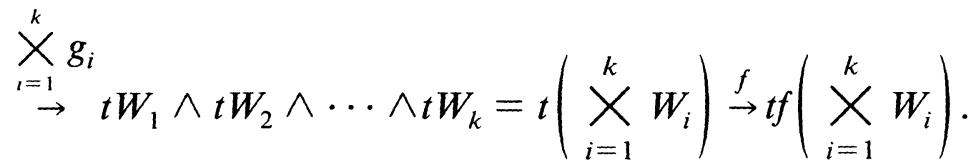

Here $\wedge$ is the smash product. This is compatible with the $H$ action and with passage to the limit.

(c) The action $\mathscr{K}(k) \times K^{k} \rightarrow K$ is induced from the natural maps

$$
\mathscr{K}(k, W) \times \mathscr{K}\left(j_{1}, W\right) \times \cdots \times \mathcal{K}\left(j_{k}, W\right) \rightarrow \mathscr{K}\left(\sum_{i=1}^{k} j_{i}, W\right)
$$

given by taking $f_{t} \in \mathscr{K}(k, W), g_{t}^{l} \in \mathcal{K}\left(j_{i}, W\right)$ and constructing a homotopy $\sum_{i=1}^{k} j_{i} \times W \rightarrow W$ defined on the subspace $j_{i} \times W$ by $j_{i} \times$ $W \stackrel{g_{t}^{\prime}}{\rightarrow} W \stackrel{f_{l} i \times W}{\rightarrow} W$. This is compatible with the $H$ action and with passage to the limit.

(d) The $\mathcal{K}(k) \times F^{k} \rightarrow F$ action is induced by maps

$$
\mathscr{K}(k, W) \times \overbrace{F(t W, t W) \times \cdots \times F(t W, t W)}^{k \text { copies }} \rightarrow F(t W, t W)
$$

given by taking $f_{t} \in \mathscr{K}(k, W)$ and $g_{i} \in F(t W, t W)$ and defining a self map of $t W$ by sending

$$
\vec{x} \rightarrow \begin{cases}\infty & \text { if } \vec{x}=\infty, \\ \infty & \text { if } \vec{x} \notin \bigcup_{i=1}^{k}\left(\left(f_{1} \mid i \times W\right) \circ g_{i}\right)(W), \\ {\left[\left(f_{1} \mid i \times W\right) \circ g_{i}\right]^{-1}(\vec{x})} & \text { otherwise. }\end{cases}
$$

This is well-defined as the $f_{1} \mid i \times W$ 's are homeomorphisms onto their images which are disjoint for different values of $i$. Thus the images of $\left(f_{1} \mid i \times W\right) \circ g_{l}(W)$ are disjoint for different values of $i$. This construction is also clearly compatible with the $H$ action and with passage to the limit. 
Furthermore it is clear that all four actions defined above commute with the configuration map $\psi$.

We close this section by noting that the action defined in part (b) above is easily modified to show $\mathcal{L}$ also acts naturally on the $H$ space $G=\lim _{\rightarrow} G(S W, S W)$, the space of free self maps of the unit sphere of $W$ where $W$ runs over all finite dimensional subspace of $V_{H}$, and $\mathcal{L}^{H}$ thus acts naturally on $G^{H}=G(H)$. This implies

THEOREM 2.2.7. The radial extension map

$$
\rho: G(H) \rightarrow F(H)
$$

is an $\mathfrak{L}^{H}$ map.

2.3. We now identify the $\varrho^{H}$ action on $F(H)$ and $G(H)$ with the composition product. We say an $\mathcal{L}^{H}$ space structure on a space $X$ refines a given $H$-space structure $\mu$ on $X$ if for each point $p \in \mathcal{L}^{H}(k)$ the following diagram commutes up to homotopy:

$$
\begin{array}{ccc}
X^{k} & k \text { fold product by } \mu & X \\
\swarrow \text { id } & & \nearrow \gamma_{\mathcal{E}^{H}} \\
p \times X^{k} & \rightarrow & \varrho^{H}(k) \times X^{k}
\end{array}
$$

The spaces $S G(H)$ and $S F(H)$ of degree 1 maps are endowed under $\varrho^{H}$ with an infinite loop space structure. Recall the standard smash product gives the composition product infinite loop space structure.

THEOREM 2.3.1. The $\mathfrak{L}^{H}$ structure on $G(H)$ and $F(H)$ refines the composition product structure. Hence $\rho: S G(H) \rightarrow S F(H)$ is an infinite loop map with respect to the composition product.

Proof. The first statement for $F(H)$ is immediate from the proof 2.2.4 part b. Minor modification gives the first statement for $G(H)$. The second statement follows immediately from the commutative diagram:

$$
\begin{array}{ccc}
\varrho^{H}(k) \times G(H)^{k} & \rightarrow & G(H) \\
\downarrow \text { id } \times \rho^{k} & & \downarrow \rho \\
\varrho^{H}(k) \times F(H)^{K} & \rightarrow & F(H)
\end{array}
$$




\section{Chapter III}

In this chapter we relate the configuration map $\phi: K^{H} \rightarrow F(H)$ to the radial extension map $\rho: G(H) \rightarrow F(H)$.

Using equivariant transversality Segal [27], and Hauschild [8], independently proved the following theorem.

THEOREM 3.1.1. The configuration map $\phi: K^{H} \rightarrow F(H)$ is a group completion.

Corollary 3.1.2. The map $\Phi_{*}: H_{*}\left(K^{H}\right) \rightarrow H_{*}(F(H))$ becomes an isomorphism after inverting $H_{0}\left(K^{H}\right)$ and for field coefficients $\Phi_{*}$ is a Hopf algebra isomorphism under both the loop sum ( $\mathscr{K}^{H}$ structure) and the composition product ( $\mathrm{E}^{H}$ structure) Pontrjagin product. Furthermore, the following diagrams commute (again with field coefficients):

$$
\begin{array}{ccc}
H_{*}\left(K^{H}\right) & \stackrel{Q^{\prime}}{\rightarrow} & H_{*}\left(K^{H}\right) \\
\downarrow \Phi_{*} & & \downarrow \Phi_{*} \\
H_{*}(F(H)) & \stackrel{Q^{\prime}}{\rightarrow} & H_{*}(F(H))
\end{array}
$$

and

$$
\begin{array}{ccc}
H_{*}\left(K^{H}\right) & \stackrel{\hat{Q}^{\prime}}{\rightarrow} & H_{*}\left(K^{H}\right) \\
\downarrow \Phi_{*} & & \downarrow \Phi_{*} \\
H_{*}(F(H)) & \stackrel{\hat{Q}^{\prime}}{\rightarrow} & H_{*}(F(H))
\end{array}
$$

So far we have discussed mainly $F(H)$, the space of semi-free or based $H$-equivariant maps for the simple reason that it is, as we have seen, well approximated by $K^{H}$. However, for geometric reasons, we are really interested in $G(H)$, the space of free $H$-equivariant maps. In this section we explain how $G(H)$ sits in $F(H)$ under $\rho$. 
TheOREM 3.1.3. The Becker-Schultz and Segal homotopy equivalences of 1.1.5 and 1.1.6 are related by the commutative diagram

$$
G(H) \stackrel{\gamma_{B S}}{\rightarrow} Q\left(B H^{+}\right)
$$

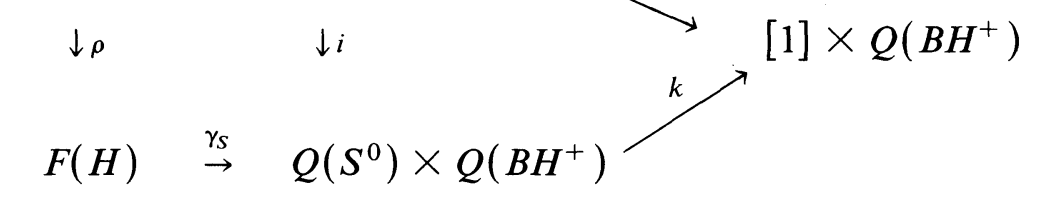

where $\gamma_{B S}$ and $\gamma_{S}$ are the Becker-Schultz and Segal equivalences, $j$ the inclusion on the second factor, $k$ the standard inclusion, and $i=k \circ j$. Recall [1] $\in Q_{1}\left(S^{0}\right)$ is the limit of $t\left(\mathbf{R}^{n}\right) \stackrel{\text { id }}{\rightarrow} t\left(\mathbf{R}^{n}\right)$.

Although 3.1.3 describes $\rho$ quite well, the theorem is not sufficient for our purposes. More precisely, although a group completion of $K^{H}$ is well-known to be homotopy equivalent, as an infinite loop space under loop sum, to $Q\left(S^{0}\right) \times Q\left(B H^{+}\right)$we must check that this equivalence is compatible with the Segal equivariant transversality map $\gamma_{S}$. The following diagram summarizes the situation.

$$
\begin{aligned}
& \text { (3.1.5) } \downarrow \gamma_{B S} \quad \downarrow \gamma_{S} \quad \downarrow \text { group complete } \\
& Q\left(B H^{+}\right) \stackrel{i}{\rightarrow} Q\left(S^{0}\right) \times Q\left(B H^{+}\right) \stackrel{\text { id }}{\rightarrow} Q\left(S^{0}\right) \times Q\left(B H^{+}\right)
\end{aligned}
$$

THEOREM 3.1.6. The diagram 3.1.5 commutes up to homotopy.

In the appendix we prove 3.1.3 and 3.1.6.

\section{CHAPTER IV}

4.1. We begin this chapter by describing the action map on $K^{H}$ and thus on a group completion $Q\left(S^{0}\right) \times Q\left(B H^{+}\right)$. Recall from [12], there is an action map
$\bar{\lambda}: B(C) \times K^{H} \rightarrow K^{H}$

induced by the homomorphism $\alpha: C \times H \rightarrow H$ defined on the group level by $\alpha(\theta, h)=\theta \cdot h$ (here $C$ is center of $H$ ). 
THEOREM 4.1.2. $\bar{\lambda}$ is described by the composition

$$
\begin{aligned}
& B C \times Q\left(S^{0}\right) \times Q\left(B H^{+}\right) \stackrel{\text { shuffle }}{\rightarrow} Q\left(S^{0}\right) \times B C \times Q\left(B H^{+}\right) \\
& \stackrel{\mathrm{id} \times \wedge}{\rightarrow} Q\left(S^{0}\right) \times Q\left((B C \times B H)^{+}\right) \stackrel{\operatorname{id} \times Q(B \alpha)}{\rightarrow} Q\left(S^{0}\right) \times Q\left(B H^{+}\right) .
\end{aligned}
$$

Proof. $\bar{\lambda}$ is a map of $\mathscr{K}^{H}$ spaces. Furthermore, $\bar{\lambda}$ is the infinite loop extension of the two maps

$$
B C \times S^{0} \times \mathrm{pt} \stackrel{\mathrm{pr}_{2}}{\rightarrow} S^{0} \times \mathrm{pt} \hookrightarrow Q\left(S^{0}\right) \times Q\left(B H^{+}\right)
$$

and

$B C \times \mathrm{pt} \times B H \stackrel{\text { shuffle }}{\rightarrow}$ pt $\times B C \times B H \stackrel{\mathrm{id} \times B \alpha}{\rightarrow}$ pt $\times B H \hookrightarrow Q\left(S^{0}\right) \times Q\left(B H^{+}\right)$.

The first composition reflects the fact that, by definition, the action of $C$ on the trivial $H$-set $\{1\}$ is trivial, while the second composition reflects the fact that $\alpha$ gives the action of $C$ on the free $H$-set $\{H\}$.

4.2. In this section we identify the maps $\Omega \psi_{G}$ and $\bar{\lambda}$. Recall $S G(V)$ $=\lim _{n} \operatorname{Map}_{H}(S(n V), S(n V))_{\text {degree } 1}$, the degree +1 component of $G(V)$. We wish to describe the geometric twist map

$$
\psi_{G}: B C \times B S G(H) \rightarrow B S G(H)
$$

which classifies $L \otimes_{\tau} \xi-L \otimes \operatorname{dim} \xi . \psi_{G}$ loops down to

$$
\Omega \psi_{G}: B C \times S G(H) \rightarrow S G(H)
$$

and $S G(H) \simeq Q_{0}\left(B H^{+}\right)[2]$.

THEOREM 4.2.3. The following diagram commutes up to homotopy:

$$
\begin{array}{ccc}
B C \times S G(H) & \stackrel{\Omega \psi_{G}}{\rightarrow} & S G(H)=Q_{0}\left(B H^{+}\right) \\
\downarrow & & \nearrow Q(B \alpha) \\
B C \times Q_{0}\left(B H^{+}\right) & \stackrel{\rightarrow}{ } & Q\left((B C \times B H)^{+}\right)
\end{array}
$$


THEOREM 4.2.4. $\Omega \psi_{G}$ extends to $\Omega \psi_{F}: B C \times F(H) \rightarrow F(H)$ which commutes with the $\mathcal{K}^{H}$ loop space structure on $F(H)$. Furthermore, the following diagram commutes:

$$
\begin{array}{ccc}
B C \times S G(H) & \stackrel{\Omega \psi_{G}}{\rightarrow} & S G(H) \\
\downarrow & & \downarrow \rho \\
B C \times F(H) & \stackrel{\Omega \psi_{F}}{\rightarrow} & F(H)
\end{array}
$$

Given the identifications of Chapter III, we may rewrite 4.2.5 as

$$
\begin{array}{ccc}
B C \times\left([1] \times Q_{0}\left(B H^{+}\right)\right) & \stackrel{\Omega \psi_{G}}{\rightarrow} & {[1] \times Q_{0}\left(B H^{+}\right)} \\
\downarrow & & \downarrow \\
B C \times Q\left(S^{0}\right) \times Q\left(B H^{+}\right) & \stackrel{\Omega \psi_{F}}{\rightarrow} & Q\left(S^{0}\right) \times Q\left(B H^{+}\right)
\end{array}
$$

Theorem 4.2.3 follows from 4.2.4 and the following lemma.

LEMMA 4.2.7. The following diagram commutes up to homotopy:

$$
\begin{array}{ccc}
B C \times Q\left(S^{0}\right) \times Q\left(B H^{+}\right) & \stackrel{\Omega \psi_{F}}{\rightarrow} & Q\left(S^{0}\right) \times Q\left(B H^{+}\right) \\
\downarrow \text { shuffle } & & \uparrow \text { id } \times Q(B \alpha) \\
Q\left(S^{0}\right) \times B C \times Q\left(B H^{+}\right) & \stackrel{\text { id } \times \wedge}{\rightarrow} & Q\left(S^{0}\right) \times Q\left((B C \times B H)^{+}\right)
\end{array}
$$

In turn, to prove 4.2 .7 we need only show

\section{LEMMA 4.2.8.}

(a) $\Omega \psi_{F}$ restricted to $B C \times S^{0} \times \mathrm{pt}$ is given by the composition

$$
B C \times S^{0} \times \mathrm{pt} \stackrel{\mathrm{pr}_{2}}{\rightarrow} S^{0} \times \mathrm{pt} \hookrightarrow Q\left(S^{0}\right) \times Q\left(B H^{+}\right) .
$$

(b) $\Omega \psi$ restricted to $B C \times \mathrm{pt} \times B H$ is given by the composition

$$
B C \times B H \stackrel{\text { shuffle }}{\rightarrow} \text { pt } \times B C \times B H \stackrel{\mathrm{id} \times B \alpha}{\rightarrow} \text { pt } \times B H \hookrightarrow Q\left(S^{0}\right) \times Q\left(B H^{+}\right) .
$$

We begin the proofs of 4.2 .4 and 4.2 .8 by giving an explicit model for $\Omega \Psi_{G}$. Let $(E C)_{k}=C * C * \cdots * C$ be the $k+1$ fold join of $C$ and $(B C)_{k}=(E C)_{k} / C$. Since $C$ is finite, the $H$ bundle $\xi=V \times_{Z}(E C)_{k}$ over $(B C)_{k}$ has finite order, say $M_{k}$; that is, $M_{k} \xi \stackrel{\mathscr{K}}{\simeq} M_{k}\left(V \times(B C)_{k}\right)$ as a $H$ bundle. This gives a $C$ equivariant map $\phi:(E C)_{k} \rightarrow \operatorname{Isom}_{H}\left(M_{k} V, M_{k} V\right)$ 
with $\mathscr{K}([x, h])=(\phi(y) x,[y])$ for $(x, y)$ in $V \times(E C)_{k}$. Here $C$ acts by premultiplication on $\operatorname{Isom}_{H}(-,-)$.

Given $N$ we get, by direct sum, a map $N \phi:(E C)_{k} \rightarrow$ Isom $_{H}\left(M_{k}(N V), M_{k}(N V)\right)$ which induces a $C$-equivariant map

$$
\widehat{N \phi}:(E C)_{k} \rightarrow \operatorname{Isom}_{H}\left(S\left(M_{k}(N V)\right), S\left(M_{k}(N V)\right)\right) .
$$

Given $f \in \operatorname{Map}_{H}(S(N V), S(N V))$ and $\alpha \in(E C)_{k}$ we consider the composition

$$
\begin{aligned}
& S\left(M_{k}(N V)\right) \stackrel{\widehat{N \phi}(\alpha)}{\rightarrow} S\left(M_{k}(N V)\right)=S(N V) * S\left(M_{k}-1\right)(N V) \\
& \stackrel{f * \text { id }}{\rightarrow} S(N V) * S\left(\left(M_{k}-1\right)(N V)\right)=S\left(M_{k}(N V)\right) \\
& \stackrel{[\widehat{N \phi}(\alpha)]^{-1}}{\rightarrow} S\left(M_{k}(N V)\right)
\end{aligned}
$$

which is well-defined as $\widehat{N \phi}(\alpha)$ is an isometry. Furthermore, as $C$ is the center of $H$, this composition is independent of the coset of $\alpha \in(E C)_{k}$ under the $C$ action. Thus we have

Definition 4.2.9. We define the map

$$
\begin{aligned}
\lambda_{k N}^{1}: & (B C)_{k} \times \operatorname{Map}_{H}(S(N V), S(N V)) \\
& \rightarrow \operatorname{Map}_{H}\left(S\left(M_{k}(N V)\right), S\left(M_{k}(N V)\right)\right)
\end{aligned}
$$

by the formula

$$
\lambda_{k N}^{1}([\alpha], f)=[\widehat{N \phi}(\alpha)]^{-1} \circ(f * \mathrm{id}) \circ[\widehat{N \phi}(\alpha)] .
$$

Passing to the limit over $N$ gives

DEFINITION 4.2.10.

$$
\lambda_{k}^{1}=\lim _{\vec{N}} \lambda_{k N}^{1}:(B C)_{k} \times G(H) \rightarrow G(H) .
$$

Thus by making choices we may pass to the limit over $k$ to obtain

DEFINITION 4.2.11.

$$
\lambda^{1}=\lim _{\vec{k}} \lambda_{k}^{1}: B C \times G(H) \rightarrow G(H)
$$

which induces $S \lambda^{1}: B C \times S G(H) \rightarrow S G(H)$. 
In $\S 4.3$ we prove

THEOREM 4.2.12. $S \lambda^{1}=\Omega \psi_{G}$.

Assuming 4.2.12 we procede with the proofs of 4.2.4 and 4.2.8. We define $N \phi^{1}:(E C)_{k} \rightarrow \operatorname{Isom}_{H}\left(M_{k}(N(\mathbf{R} \oplus V)), M_{k}(N(\mathbf{R} \oplus V))\right)$ by the assignment $\alpha \rightarrow(N \phi)(\alpha)$ on $M_{k}(N V)$ and $\alpha \rightarrow$ id on $M_{k}(N \mathbf{R})$. Note that $N \phi^{1}$ is $C$ equivariant. Thus in a manner similar to 4.2 .9 and 4.2.10, we obtain

DeFinition 4.2.13. The map

$$
\lambda_{k}^{11}:(B C)_{k} \times F(H) \rightarrow F(H)
$$

is induced by the maps

$$
\lambda_{k, N}^{11}([\alpha], f)=\left[t\left(N \phi^{1}(\alpha)\right)\right]^{-1} \circ(f \wedge \mathrm{id}) \circ\left[t\left(N \phi^{1}(\alpha)\right)\right]
$$

on the finite levels.

Notice, by 4.2.11, 4.2.12 and 4.2.13, that 4.2.5 commutes. Also note that for $g \in \operatorname{Map}_{H}(t(N \mathbf{R}), t(N \mathbf{R}))=\operatorname{Map}(t(N \mathbf{R}), t(N \mathbf{R}))$ that the suspension $g$,

$$
g \wedge \mathrm{id} \in \operatorname{Map}_{H}(t(N(\mathbf{R} \oplus V)), t(N(\mathbf{R} \oplus V))),
$$

has the property $\lambda_{k N}^{11}([\alpha], g \wedge$ id $)=(g \wedge$ id $) \wedge$ id since " $C$ does not twist $g \wedge$ id at all." This uses the fact that the $H$ action on $N \mathbf{R}$ is trivial. Thus, under the identification $F(H) \simeq Q\left(S^{0}\right) \times Q\left(B H^{+}\right)$the following diagram commutes:

$$
\begin{aligned}
& (B C)_{k} \times Q\left(S^{0}\right) \times \mathrm{pt} \quad \Rightarrow \quad(B C)_{k} \times Q\left(S^{0}\right) \times Q\left(B H^{+}\right) \\
& \downarrow \mathrm{pr}_{2} \quad \downarrow \lambda_{k}^{1} \\
& Q\left(S^{0}\right) \times \mathrm{pt} \quad \Rightarrow \quad Q\left(S^{0}\right) \times Q\left(B H^{+}\right)
\end{aligned}
$$

Along with 4.2.12 this proves 4.2.8a.

Proposition 4.2.15. The following diagram commutes up to homotopy

$$
\begin{array}{rlrl}
(B C)_{k} & \times \mathcal{K}^{H}(j) \times F(H)^{(j)} & \stackrel{\operatorname{id} \times \gamma_{\Re^{H}}}{\rightarrow} & (B C)_{k} \times F(H) \\
& \downarrow(\text { shuffle }) \circ\left(\Delta^{j} \times \text { id } \times \text { id }\right) & & \\
\mathcal{K}^{H}(j) & \times\left[(B C)_{k} \times F(H)\right]^{(j)} & & \\
& \downarrow \lambda_{k}^{11} \\
\mathscr{K}^{H}(j) \times\left(\lambda_{k}^{11}\right)^{(j)} & \times F(H)^{(j)} \stackrel{F(H)}{\gamma_{\mathscr{K}^{H}}} &
\end{array}
$$


Proof. [12.2.6].

Of course, we may stabilize 4.2 .15 over $k$ and obtain a similar commutative diagram for $\lambda^{11}$. This finishes the proof of 4.2.4. We conclude this section by proving $4.2 .8 \mathrm{~b}$. We must check the commutivity of the diagram up to homotopy:

$$
\begin{array}{lcc}
B C \times \mathrm{pt} \times B H & \stackrel{\text { id } \times i}{\rightarrow} & B C \times F(H) \\
\quad \downarrow \text { shuffle } & & \lambda^{11} \\
\text { pt } \times B C \times B H & & \\
\quad \text { id } \times B \alpha & & \downarrow \\
\text { pt } \times B H & \stackrel{i}{\rightarrow} & F(H)
\end{array}
$$

The map

$$
i: B H=\underset{\vec{N}}{\lim } \operatorname{Map}_{H}\left(\{H\}, N(\mathbf{R} \oplus V)-N(\mathbf{R} \oplus V)^{H}\right) / H \rightarrow F(H)
$$

is given by the configuration map which sends

$$
f \in \operatorname{Map}_{H}\left(\{H\}, N(\mathbf{R} \oplus V)-N(\mathbf{R} \oplus V)^{H}\right)
$$

to the $\frac{1}{2} \varepsilon_{f}$-ball map associated to $f(H) \subset N(\mathbf{R} \oplus V)$ where $\varepsilon_{f}$ is the minimum distance between elements of $f(H)$. It is easy to check, as $\lambda_{k N}^{11}$ maps the image of $i_{N}$ to the image of $i_{M_{k} N}$, that we have a commutative diagram

$$
\begin{aligned}
& (B C)_{k} \times \operatorname{Map}_{H}\left(\{H\}, N(\mathbf{R} \oplus V)-N(\mathbf{R} \oplus V)^{H}\right) \stackrel{\mathcal{K}_{k N}^{\prime \prime}}{\rightarrow} \quad \operatorname{Map}_{H}\left(\{H\}, M_{k} N(\mathbf{R} \oplus V)-M_{k} N(\mathbf{R} \oplus V)^{H}\right) \\
& \downarrow \text { id } \times_{\varepsilon} \text { ball map } \quad \downarrow \varepsilon \text { ball map } \\
& (B C)_{k} \times \operatorname{Map}_{H}(t(N(\mathbf{R} \oplus V)), t(N(\mathbf{R} \oplus V))) \quad \stackrel{\lambda_{\sharp}^{\prime \prime N}}{\rightarrow} \quad \operatorname{Map}_{H}\left(t\left(M_{k} N(\mathbf{R} \oplus V)\right), t\left(M_{k} N(\mathbf{R} \oplus V)\right)\right)
\end{aligned}
$$

which is compatible with limits. This implies

$$
\begin{array}{ccc}
(B C)_{k} \times B H & \stackrel{\lambda_{k}^{\prime \prime}}{\rightarrow} & B H \\
\downarrow \text { id } \times i & & \downarrow i \\
(B C)_{k} \times F(H) & \stackrel{\lambda_{k}^{\prime \prime}}{\rightarrow} & F(H)
\end{array}
$$


also commutes. Thus it suffices to note:

Proposition 4.2.18. On the level of fundamental groups $\hat{\lambda}_{k}^{11}$ is precisely the map $\alpha: C \times H \rightarrow H$ given by $\alpha(\theta, h)=\theta \cdot h$.

Proof. This is a simple consequence of lifting the map $\lambda_{k}^{11}$ to the universal cover $(E C)_{k} \times E H \rightarrow E H$ and looking over a point to see the action $C \times H \rightarrow H$.

This completes the proof of $4.2 .8 \mathrm{~b}$ and therefore 4.2.3.

4.3. We conclude this chapter by proving 4.2.12. That is, we must deloop $\psi_{G}: B C \times B S G(H) \rightarrow B S G(H)$. We first require a model for $\psi_{G}$ itself. Let $\eta_{N} \rightarrow B S G(N V)$ be the universal $S G(N V)$ bundle. We may choose an open cover $\left\{U_{\alpha}\right\}$ trivializing $\eta_{N}$ and thus obtain cocycles $g_{\alpha, \beta}$ : $\left\{U_{\alpha} \cap U_{\beta} \rightarrow S G(N V)\right\}$ for $\eta_{N}$. Now form the bundles $\xi_{\alpha}$ over $B C \times U_{\alpha}$ where $\xi_{\alpha}=S G(N U) \times{ }_{C} E C \times U_{\alpha}$. As $C$ is the center of $H$ we may glue $\xi_{\alpha} \mid U_{\alpha} \cap U_{\beta}$ to $\xi_{\beta} \mid U_{\alpha} \cap U_{\beta}$ by the self-map $[x, y, z] \rightarrow\left[g_{\alpha \beta}(z), x, y, z\right]$ on $S G(N V) \times{ }_{C}(E C)_{k} \times\left(U_{\alpha} \cap U_{\beta}\right)$. This defines a twisted bundle $\tilde{\eta}_{N} \rightarrow B C$ $\times B S G(N V)$ and so defines a map $\bar{\lambda}_{N}: B C \times B S G(N V) \rightarrow B S G(N V)$.

In order to stabilize we take the trivial $S G(N V)$ bundle $[N V]=$ $S G(N V) \times B S G(N V)$ and twist it to give $[\widetilde{N V}]_{N}$ over $B C \times B S G(N V)$. Now if we classify the difference $\tilde{\eta}_{N}-[\widetilde{N V}]_{N}$, we may stabilize to yield the geometric twist map $\psi_{G}: B C \times B S G(H) \rightarrow B S G(H)$. Now as $\psi_{G}$ sends $B C \times \mathrm{pt}$ to pt we may deloop $\psi_{G}$. Consider the composition

$$
B C \times\left(S^{1} \wedge S G(N V)^{+}\right) \Rightarrow B C \times B S G(N V) \stackrel{\bar{\lambda}_{N}}{\rightarrow} B S G(N V) .
$$

$S^{1} \wedge S G(N V)^{+}$is the union of an upper cone $C_{+} S G(N V)$ and a lower cone $C_{-} S G(N V)$ and $\left.\tilde{\eta}\right|_{S^{\prime} \wedge S G(N V)^{+}}$may be described by the clutching function $C_{+} S G(N V) \cap C_{-} S G(N V)=S G(N V) \stackrel{\text { id }}{\rightarrow} S G(N V)$. Hence we may describe $\tilde{\eta}_{N} \mid B C \times S^{1} \times S G(N V)$ by clutching $\xi_{+}=S(N V) \times{ }_{C} E C$ $\times C_{+} S G(N V)$ over $B C \times C_{+} S G(N V)$ to $\xi_{-}=S(N V) \times{ }_{C} E C \times$ $C_{-}(N V)$ over $B C \times C_{-} S G(N V)$ via $[x, y, z] \rightarrow[z \circ x, y, z]$ over $B C \times$ $S G(N V)$.

But over $(B C)_{k}$ we have a trivialization $\left\{N V \oplus\left(M_{k}-1\right) N V \times_{C}(E C)_{k}\right\} \cong\left\{N V \oplus\left(M_{k}-1\right) N V \times(B C)_{k}\right\}$ 
of $H$ bundles where $M_{k}$ is sufficiently large. Hence over $(B C)_{k} \times$ $B S G(N V)$ we have

$$
\begin{aligned}
{[\widetilde{N V}]_{N} \otimes } & {\left[\left(M_{k}-1\right) N V\right]_{n} \sim S G\left(N V \oplus\left(M_{k}-1\right) N V\right) } \\
& \times B C_{k} \times B S G(N V)
\end{aligned}
$$

where $\otimes$ means fibre-wise joint and $\sim$ means homeomorphism. Thus a model for

$$
\tilde{\eta}_{N}-[\widetilde{N V}]_{N} \mid(B C)_{k} \times B S G(n V)
$$

is

$$
\tilde{\eta}_{N} \mid(B C)_{k} \otimes\left[{\widetilde{\left(M_{k}-1\right) N V}}_{N} .\right.
$$

Thus we have proved

THEOREM 4.3.2. $\tilde{\eta}_{N}-[\widetilde{N V}]_{N}$ restricted to $(B C)_{k} \times\left[S^{1} \wedge S G(N V)\right]$ may be obtained by identifying

$$
\left\{\begin{array}{c}
S G\left(M_{k} N V\right) \times(E C)_{k} \times C_{+} S G(N V) \\
\downarrow \\
(E C)_{k} \times C_{+} S G(N V)
\end{array}\right\}
$$

and

$$
\left\{\begin{array}{c}
S G\left(M_{k} N V\right) \times(E C)_{k} \times C_{-} S G(N V) \\
\downarrow \\
(E C)_{k} \times C_{-} S G(N V)
\end{array}\right\}
$$

along $(E C)_{k} \times S G(N V)$ via the identification

$$
(x, y, w) \sim\left(\left(\left.w * \mathrm{id}\right|_{S\left(M_{k}-1\right) N V}\right) \circ x, y, w\right)
$$

and then dividing out by the free action of $C$ given by $\sigma \cdot(x, y, w)=$ $\left(\sigma \cdot x, \sigma^{-1} y, w\right)$ on each piece.

Here the action of $C$ on $S G\left(M_{k} N V\right)$ is given by precomposition. To prove 4.2.12 we merely reinterpret 4.3.2.

THEOREM 4.3.3. $\tilde{\eta}_{N}-[\widetilde{N V}]_{N}$ restricted to $(B C)_{k} \times\left[S^{1} \wedge S G(N V)\right]$ may be obtained by identifying

$$
\left\{\begin{array}{c}
S G\left(M_{k} N V\right) \times(B C)_{k} \times C_{+} S G(N V) \\
\downarrow \\
(B C)_{k} \times C_{+} S G(N V)
\end{array}\right\}
$$


and

$$
\left\{\begin{array}{c}
S G\left(M_{k} N V\right) \times(B C)_{k} \times C_{-} S G(N V) \\
\downarrow \\
(B C)_{k} \times C_{-} S G(N V)
\end{array}\right\}
$$

along $(B C)_{k} \times S G(N V)$ via the identification

$$
(x,[y], w) \sim\left([N \phi(y)]^{-1} \circ\left(w \times\left.\mathrm{id}\right|_{S\left(M_{k}-1\right) N V}\right) \circ x \circ[\widehat{N \phi}(y)],[y], w\right) .
$$

Proof. $M_{k}(N V) \times_{C}(E C)_{k}$ is a trivial $H$ bundle.

As a self-map on $S G\left(M_{k} N V\right) \times(B C)_{k} \times C_{+} S G(N V)$ given by

$$
(x,[y], w) \rightarrow\left([\widehat{N \phi}(y)] \circ x \circ[\widehat{N \phi}(y)]^{-1},[y], w\right)
$$

is an identification over $(B C)_{k} \times C_{+} S G(N V)$, we may modify 4.3 .3 by replacing the last identification by

$$
(x,[y], w) \sim\left([\widehat{N \phi}(y)]^{-1} \circ\left(w \times\left.\mathrm{id}\right|_{S\left(M_{k}-1\right) N V}\right) \circ[\widehat{N \phi}(y)] \circ x,[y], w\right) .
$$

That is, $\tilde{\boldsymbol{\eta}}_{N}-[\widetilde{N V}]_{N}$ restricted to $(B C)_{k} \times\left(S^{1} \wedge S G(N V)^{+}\right)$pulls back from $S^{1} \wedge\left[(B C)_{k} \times S G(N V)\right]^{+}$and is obtained from the clutching map $\lambda_{k N}^{1}:(B C)_{k} \times S G(N V) \rightarrow S G\left(M_{k} N V\right)$ of 4.2.9. Thus $\lambda_{k N}^{1}$ is the desired adjoint of

$$
(B C)_{k}^{+} \wedge S^{1} \wedge S G(N V)^{+} \rightarrow(B C)_{k}^{+} \wedge B S G(H) \stackrel{\psi_{G}}{\rightarrow} B S G(H) .
$$

This establishes 4.2.12.

\section{ChAPTER V}

5.1. Let $H=\mathbf{Z} / p^{n}$ for some prime $p$ and integer $n$. It remains to prove the results of Chapter I. $S G(H)$ and $S F(H)$, the spaces of degree 1 maps in $G(H)$ and $F(H)$ respectively, are infinite loop spaces with respect to the composition product. To compute

$$
H_{*}(S G(H), \mathbf{Z} / p), H_{*}(B S G(H), \mathbf{Z} / p)
$$

and all related transfer maps it suffices, by the results of Chapter III to compute

$$
H_{*}(S \bar{G}(H), \mathbf{Z} / p), H_{*}(B S \bar{G}(H), \mathbf{Z} / p)
$$


and all the related transfer maps where $S \bar{G}(H)$ is the factor identified with [1] $\times Q_{0}\left(B H^{+}\right) \subset K^{H}$. The specific calculations on [1] $\times Q_{0}\left(B H^{+}\right)$ we require were carried out in [12]. Summarizing the facts we need from [6], [22], and [12] to compute we recall:

1. The composition product and composition Dyer-Lashof operations, - and $\tilde{Q}^{t}$, as well as the loop sum and loop Dyer-Lashof operations, * and $Q^{t}$, are related by the usual Cartan and Adem formulae.

2. The loop and composition products are related by the mixed Cartan formula and the distributivity formula $(x * y) \circ z=\Sigma \pm$ $\left(x \circ z^{\prime}\right) *\left(y \circ z^{\prime \prime}\right)$ where $\Delta z=\sum z^{\prime} \otimes z^{\prime \prime}$.

3 . We have the following May formula, which we state, for convenience, when $p=2$ :

$$
Q^{s}(y) \circ z=\sum Q^{s+1}\left(y \circ S q_{*}^{i} z\right) .
$$

See [6] for the odd prime analog.

4. The composition product $\circ$ on $K^{H} \simeq Q\left(S^{0}\right) \times Q\left(B H^{+}\right)$extends the diagonal transfer map \#: $Q\left(B(H \times H)^{+}\right) \rightarrow Q\left(B H^{+}\right)$induced by the diagonal inclusion $\Delta: H \rightarrow H \times H$. A result of Schultz, [22] (see also [13]), allows the computation of \# on the cells of $B H^{+}$as follows:

THEOREM 5.1.1 [22].

$$
\#\left(e_{r}, e_{s}\right)=\sum_{i=0}^{r} e_{r-\imath} \wedge t\left(\mu_{*}\left(\chi e_{\imath} \otimes e_{s}\right)\right),
$$

where $\chi$ is the canonical antiautomorphism, $\mu$ is the group multiplication map $M: H \times H \rightarrow H$, and $t$ is the transfer induced by the inclusion $1 \hookrightarrow H$.

We remark that 5.1.1 is true for all abelian compact Lie groups ([13]).

5. The loop sum, loop Dyer-Lashof, and transfer calculations of [12] apply.

We note that the results of $\S 1.3$ follow immediately from Chapter III and [12]. Now the results of [12] on the transfers, 5.1.1, and the remarks 1 through 5 above make the remaining computations in this section purely formal. To make these calculations we require a weight filtration a la May [6], and Tsuchiya [33]. Let $n \geq 1$.

DeFINITION 5.1.2. [13]. The weight valuation

$$
w: H_{*}\left(Q\left(B \mathbf{Z} / p^{n^{+}}\right), \mathbf{Z} / p\right) \rightarrow N
$$


where $N$ is the set of nonnegative integers is the smallest function with the stated domain and range satisfying

$$
\begin{gathered}
w(x * y) \geq w(x)+w(y), \\
w(x+y) \geq \min \{w(x), w(y)\}, \\
w\left(Q^{I}\left(e_{r}\right)\right) \geq p^{l(I)+1} \quad \text { for } e(I)>r \geq 0, l(I)>0, \\
w\left(e_{r}\right)=p \text { for } r>0, \\
w\left(e_{0}\right)=0
\end{gathered}
$$

where $I$ is an admissible sequence.

Let $\bar{X}_{I, \alpha}=Q^{I}\left(e_{\alpha}\right)$ where we allow $l(I)=0$ in which case $\bar{X}_{I, \alpha}=e_{\alpha}$. We now state our main technical result (compare with [18], [6], and [33]).

LEMMA 5.1.3. In any $\left(H_{*}\left(K^{\mathbf{Z} / p^{n}}\right), \mathbf{Z} / p\right), n \geq 1$,

$$
\bar{X}_{I, \alpha} \circ \bar{X}_{J, \beta}=\bar{X}_{I, \alpha} * \bar{X}_{J, \beta}
$$

modulo elements of higher weight where:

(a) Either $l(I)$ or $l(J)$ is greater than zero if $p=2$.

(b) If $p>2$ there is no restriction on $l(I)$ or $l(J)$.

We require the following two computational lemmas. We may simultaneously consider $\mathbf{Z} / p^{n}$ for all $p$ and $n \geq 1$.

LEMMA 5.1.4. $w\left(Q^{I}\left(e_{\alpha}\right) \circ Q^{J}\left(e_{\beta}\right)\right) \geq p^{l(I)+l(J)+2}$.

Proof. First assume $\alpha, \beta>0$. By May's formula

$$
Q^{I}\left(e_{\alpha}\right) \circ Q^{J}\left(e_{\beta}\right)=\sum_{I^{\prime}, J^{\prime}, \alpha^{\prime}, \beta^{\prime}} Q^{I^{\prime}} Q^{J^{\prime}}\left(e_{\alpha^{\prime}} \circ e_{\beta^{\prime}}\right)
$$

where $l(I)=l\left(I^{\prime}\right), l(J)=l\left(J^{\prime}\right)$ and $\alpha^{\prime}, \beta^{\prime}>0$. But 5.1.1, 1.3.3 and 1.3.5 clearly show $w\left(e_{\alpha^{\prime}} \circ e_{\beta^{\prime}}\right) \geq p^{2}$. Thus the lemma follows for $\alpha . \beta>0$. The other cases are similar and left to the reader.

LEMMA 5.1.5. $w\left(Q^{I}\left(e_{\alpha}\right) \circ \chi\left(e_{0}^{p^{m}}\right)\right) \geq p^{l(I)+1+m+1}$.

Proof. $Q^{I}\left(e_{\alpha}\right) \circ \chi\left(e_{0}^{p^{m}}\right)=Q^{I}\left(e_{\alpha} \circ \chi\left(e_{0}^{p^{m}}\right)\right)=\chi Q^{I}\left(e_{\alpha} \circ e_{0}^{p^{m}}\right)=$ $\chi Q^{I}\left(e_{\alpha}^{\circ}\left(p^{m} e_{0}\right)\right)=\chi\left(Q^{I^{\prime}}\left(e_{\alpha} \circ e_{0}\right)\right)^{* p^{m}}$ by the Cartan formula and distributivity. As $\chi$ preserves weight the lemma follows. 
Proof of 5.1.3. Expanding

$$
\left(Q^{I}\left(e_{\alpha}\right) *\left([1] * \chi\left(e_{0}^{p^{(I)}}\right)\right)\right) \circ\left(Q^{J}\left(e_{\beta}\right) *\left([1] * \chi\left(e_{0}^{p^{(J)}}\right)\right)\right)
$$

we obtain

$$
\begin{aligned}
& \sum_{I^{\prime}, J^{\prime}, \alpha^{\prime}, \beta^{\prime}}\left(Q^{I^{\prime}}\left(e_{\alpha^{\prime}}\right) \circ Q^{J^{\prime}}\left(e_{\beta^{\prime}}\right)\right) *\left(Q^{I^{\prime \prime}}\left(e_{\alpha^{\prime \prime}}\right) \circ\left([1] * \chi\left(e_{0}^{p^{(J)}}\right)\right)\right) \\
& *\left(Q ^ { J ^ { \prime \prime } } ( e _ { \beta ^ { \prime \prime } } ) \circ ( [ 1 ] * \chi ( e _ { 0 } ^ { p ^ { ( I ) } } ) ) * \left([ 1 ] * \chi \left(e^{\left.\left.\left.p^{(I I)+(J)}-p^{(I)}-p^{(I)}\right)\right)\right)}\right.\right.\right.
\end{aligned}
$$

where $l\left(I^{\prime}\right)=l\left(I^{\prime \prime}\right)=l(I), l(J)=l\left(J^{\prime \prime}\right)=l(J)$ and $\alpha^{\prime}>0$ if $\alpha>0, \beta^{\prime}>$ 0 if $\beta>0$. But the two middle terms may be rewritten as

$$
Q^{I^{\prime \prime \prime}}\left(e_{\alpha^{\prime \prime \prime}}\right) *\left(Q^{I^{\prime \prime \prime \prime}}\left(e_{\alpha^{\prime \prime \prime \prime}}\right) \circ \chi\left(e_{0}^{p^{(J)}}\right)\right)
$$

and

$$
Q^{J^{\prime \prime \prime}}\left(e_{\beta^{\prime \prime \prime}}\right) *\left(Q^{J^{\prime \prime \prime \prime}}\left(e_{\beta^{\prime \prime \prime \prime}}\right) \circ \chi\left(e_{0}^{p^{(I)}}\right)\right)
$$

where 5.1.4 and 5.1.5 imply each nontrivial $\circ$ product term has weight $\geq p^{l(I)+l(J)+2}$. The lemma follows immediately from the observation that $p^{l_{1}+l_{2}+2} \geq p^{l_{1}+1}+p^{l_{2}+1}$ and equality holds only for $p=2, l_{1}=l_{2}=0$.

5.1.3 immediately implies 1.2.10. Now consider $p=2$. First let $n=1$. 1.2.5 is precisely Corollary 5.3.a of [13]. We note, in passing, that it is easy to check that $J_{\mathbf{Z} / 2}\left(e_{r}\right)^{\circ 2}=0$ by direct computation:

$$
\begin{aligned}
J_{\mathbf{Z} / 2}\left(e_{r}\right)^{\circ 2}= & \sum\left(e_{r-i} \circ\left(e_{0} *[-1]\right) \circ e_{r-j} \circ\left(e_{0} *[-1]\right)\right) \\
& *\left(\left(e_{i} \circ\left(e_{0} *[-1]\right)\right) \circ\left([1] * \chi\left(e_{0}\right)\right)\right) \\
& *\left(\left([1] * \chi\left(e_{0}\right)\right) \circ\left(e_{j} \circ\left(e_{0} *[-1]\right)\right)\right) *[1]
\end{aligned}
$$

by repeated distributivity. As in [18] we note that $i=j$ in the above sum by symmetry as we are working mod 2. But 5.1.3 and 1.3.1.a imply the sum reduces to $\Sigma Q^{r-i}\left(e_{r-i}\right) * \chi e_{i} * \chi e_{i} *[1]=\Sigma\left(\chi\left(e_{r-i}\right) * e_{i}\right)^{* 2} *[1]=$ 0 .

Proof of 1.2.4 and 1.2.6. Part (a) of 1.2.4 is trivial. We note in passing that the computation above showing $J_{\mathrm{Z} / 2}\left(e_{r}\right)^{\circ 2}=0$ and the fact that the algebra map commutes with both products would be sufficient, even without 5.3a of [13], to formally show $E\left[J_{\mathrm{Z} / 2}\left(e_{r}\right) \mid r>0\right]$ is a sub-Hopf algebra of $H_{*}(S G(\mathbf{Z} / 2), \mathbf{Z} / 2)$. Now consider the exact sequence

$$
\begin{aligned}
0 & \rightarrow H_{*}(S O, \mathbf{Z} / 2) \stackrel{J_{\mathbf{Z} / 2^{*}}}{\rightarrow} H_{*}(S G(\mathbf{Z} / 2), \mathbf{Z} / 2) \\
& \rightarrow H_{*}(S G(\mathbf{Z} / 2), \mathbf{Z} / 2) / / H_{*}(S O, \mathbf{Z} / 2) \rightarrow 0 .
\end{aligned}
$$


As we have identified the image of $J_{\mathbf{Z} / 2}\left(e_{r}\right)$ with $e_{r} *[-1]$ modulo higher weight (5.1.3), it follows that $H_{*}(S G(\mathbf{Z} / 2), \mathbf{Z} / 2) / / H_{*}(S O, \mathbf{Z} / 2)$ is a polynomial subalgebra on the stated generators of 1.2.4.b. The weight filtration, 5.1.3, and 1.2.4.b then imply 1.2.6.

Now let $n>1$. While it is not true that $\bar{e}_{2 r} \circ \bar{e}_{2 r}$ is equal to $\bar{e}_{2 r} * \bar{e}_{2 r}$ modulo higher weight, we do have the following:

LEMMA 5.1.6. $e_{2 r} \circ e_{2 r}=e_{2 r} * e_{2 r}+\Sigma_{i<2 r} Q^{4 r-i}\left(e_{i}\right) *\left([1] \circ \chi\left(e_{0}\right)^{4}\right)$ in $H_{*}\left(S G\left(\mathbf{Z} / 2^{n}\right), \mathbf{Z} / 2\right)$.

Proof.

$$
\begin{aligned}
\left(e_{2 r} *[1] * \chi\left(e_{0}\right)\right)^{\circ 2}= & \sum_{i}\left(e_{2 r-i} \circ e_{2 r-i}\right) *\left(e_{i} \circ\left([1] * \chi\left(e_{0}\right)\right)\right) * 2 \\
& *\left([1] * \chi e_{0} * \chi e_{0} * e_{0}^{2}\right)
\end{aligned}
$$

by mod 2 symmetry. This simplifies to

$$
\sum\left(e_{2 r-i} \circ e_{2 r-i}\right) *\left(e_{i-j}\right) * 2 *\left(\chi\left(e_{i} \circ e_{0}\right)\right)^{* 2} *\left([1] * \chi e_{0} * \chi e_{0} * e_{0}^{2}\right) .
$$

Thus there are three terms of possible lowest weight, namely

$$
\begin{gathered}
\left(e_{2 r} \circ e_{2 r}\right) *\left([1] * \chi\left(e_{0}^{2}\right)\right), \quad e_{2 r} * e_{2 r} *\left([1] * \chi\left(e_{0}^{2}\right)\right) \quad \text { and } \\
\left(\chi\left(e_{2 r} \circ e_{0}\right)\right) * 2 *\left(e_{0}^{2} *[-1]\right) .
\end{gathered}
$$

5.1.1 and 1.3.3 clearly show, however, that the only terms of weight $<2^{3}=8$ in $e_{2 r} \circ e_{2 r}$ and $\mathrm{e}_{2 r} \circ e_{0}$ are of the form $Q^{4 r-i}\left(e_{i}\right)$ for $i<2 r$.

\subsection{6 and a slight generalization of 5.1.3 yield}

COROLlaRY 5.1.7. $\bar{e}_{2 r}$ is a polynomial generator in $H_{*}\left(S G\left(\mathbf{Z} / 2^{n}\right), \mathbf{Z} / 2\right)$ for $n>1$.

The proof of 1.2.9 now follows from 4.4.c and 5.1.c of [13], and from 5.1.3 and 5.1.7.

We now turn to the Hopf algebra structure of the classifying spaces $B S G\left(\mathbf{Z} / p^{n}\right)$ by studying the Eilenberg-Moore classifying space spectral sequence as in [6], [5] and [13].

$$
\operatorname{Tor}_{s, t}^{H_{*}\left(S G\left(\mathbf{Z} / p^{n}\right), \mathbf{Z} / p\right)}(\mathbf{Z} / p, \mathbf{Z} / p) \Rightarrow H_{*}\left(B S G\left(\mathbf{Z} / p^{n}\right), \mathbf{Z} / p\right) .
$$


This is a first quadrant homology spectral sequence of Hopf algebras. Consider the following cases:

Case 1. $p=2, n=1$. Then $H_{*}(S O, \mathbf{Z} / 2)$ in $H_{*}(S G(\mathbf{Z} / 2), \mathbf{Z} / 2)$ yields generators in $E^{2}$, namely the divided powers of the suspension $\sigma\left(J_{\mathbf{Z} / 2}\left(e_{r}\right)\right)$ of $J_{\mathbf{Z} / 2}\left(e_{r}\right)$. As $J_{\mathbf{Z} / 2}$ maps $S O$ onto these elements and the spectral sequence

$$
\operatorname{Tor}_{s, t}^{H_{*}(S O, \mathbf{Z} / 2)}(\mathbf{Z} / 2, \mathbf{Z} / 2) \Rightarrow H_{*}(B S O, \mathbf{Z} / 2)
$$

collapses at $E^{2}$, we conclude that these generators are permanent cycles. As there are no further generators with $s>1,5.1 .8$ also collapses at $E^{2}$.

Case 2. $p=2$ and $n \geq 2$ or $p$ odd. Then $t_{n}: J_{S^{1}}: U \rightarrow S G\left(S^{1}\right) \rightarrow$ $S G\left(\mathbf{Z} / p^{n}\right)$ includes $H_{*}(U, \mathbf{Z} / p)$ in $H_{*}\left(S G\left(\mathbf{Z} / p^{n}\right), \mathbf{Z} / p\right)$ and yields generators in $E^{2}$ of 5.1 .8 , namely the divided powers of the suspensions $\sigma\left(\left(t_{n} \circ J_{S^{1}}\right)_{*} \bar{a}_{r}\right)$ of $\left(t_{n} \circ J_{S^{1}}\right)_{*} \bar{a}_{r}\left(\right.$ recall $\left(t_{n} \circ J_{S^{1}}\right)_{*} \bar{a}_{r}=J_{n}\left(e_{2 r+1}\right)$ when $p=$ 2). As $t_{n} \circ J_{S^{1}}$ maps $U$ onto these elements and the spectral sequence

$$
\operatorname{Tor}_{s, t}^{H_{*}(U, \mathbf{Z} / p)}(\mathbf{Z} / p, \mathbf{Z} / p) \Rightarrow H_{*}(B U, \mathbf{Z} / p)
$$

collapses at $E^{2}$ we again conclude that these generators are permanent cycles. Again, when $p=2$, there are no further generators with $s>1$, so the spectral sequence 5.1.8 collapses at $E^{2}$. For $p$ odd, each odd generator in $H_{*}\left(S G\left(\mathbf{Z} / p^{n}\right), \mathbf{Z} / p\right)$ yields a divided power algebra in $E^{2}$. Just as in the non-equivariant case, [6], they are connected by a universal differential, [6],

$$
d^{p-1} \gamma_{p+j}(\sigma x)=-\left(\sigma \beta \tilde{Q}_{1} x\right) \gamma_{j}(\sigma x) .
$$

(Recall that if $2 s=|x|+1$, then $\tilde{Q}_{1} x=\omega \tilde{Q}^{s} x$ for some unit $\omega \in \mathbf{Z} / p$.) To compute $\tilde{Q}_{1}$ we have

LEMMA 5.1.9. For $p$ odd

$$
\tilde{Q}_{1}\left(Q^{I}\left(e_{r}\right) *[1] *\left(\chi e_{0}\right)^{p^{(I)}}\right) \equiv Q_{1} Q^{I}\left(e_{r}\right) *[1] *\left(\chi e_{0}\right)^{p^{(I I)+1}}
$$

modulo higher weight in $H_{*}\left(S G\left(\mathbf{Z} / p^{n}\right), \mathbf{Z} / p\right)$ provided that $I$ is admissible. with $e(I)>r$ and $l(I)>0$.

Assuming the lemma, which we prove later, the $E^{p}$ term has the form

$$
\begin{aligned}
E^{p} \cong & \Gamma\left[\sigma\left(\left(t_{n} \circ J_{S^{1}}\right)_{*} \bar{a}_{r}\right): r \geq 0\right] \\
& \otimes E\left[\sigma\left(\left(t_{n} \circ J_{S^{1}}\right)_{*} \beta Q^{s} \bar{a}_{r}\right): s>r>0\right] \otimes D
\end{aligned}
$$


where $D$ is a free commutative algebra truncated at height $p$. As no further differentials are possible, $E^{p}=E^{\infty}$.

5.1.9 and the equivariant $J$ homomorphism solve the extension problems when $p$ is odd and thus yields 1.2.14.

For $p=2$ we require several lemmas to take the place of 5.1.9. However, 5.1.10 through 5.1.15 do, along with the equivariant $J$ homomorphism, solve the extension problems at 2 and thus yield 1.2.12 and 1.2.13.

LEMMA 5.1.10. In $H_{*}(S G(\mathbf{Z} / 2), \mathbf{Z} / 2)$

$$
\tilde{Q}^{r+1}\left(Q^{r}\left(e_{0}\right) *[1] * \chi e_{0}^{2}\right) \equiv Q^{r+1} Q^{r}\left(e_{0}\right) *[1] * \chi\left(e_{0}^{3}\right)
$$

modulo higher weight.

Proof. By May's formula, $Q^{r}\left(e_{0}\right)=Q^{r}[1] \circ e_{0}$. Thus

$$
\tilde{Q}^{r+1}\left(Q^{r} e_{0}\right)=\tilde{Q}^{r+1}\left(Q^{r}(1) \circ e_{0}\right)=\sum_{s=1}^{r+1} \tilde{Q}^{r}(1) \circ Q^{s}\left(e_{0}\right)
$$

but evidently the only non-zero terms in the sum are $\tilde{Q}^{r+1} Q^{r}(1) \circ Q^{0} e_{0}$ and $\tilde{Q}^{r} Q^{r}(1) \circ Q^{\prime} e_{0}$. As $Q^{0} e_{0}=e_{0} * e_{0}$ and $\tilde{Q}^{r+1} Q^{r}(1)$ is odd dimensional, the first term is zero by distributivity. As $\tilde{Q}^{r} Q^{r}(1) \circ Q^{1}\left(e_{0}\right)=$ $Q^{r}(1) \circ Q^{r}(1) \circ Q^{1}\left(e_{0}\right)$ and as $Q^{r}(1) \circ Q^{r}(1)=Q^{r}(1) * Q^{r}(1)$, [18], distributivity again implies the second is zero. Now apply the mixed Cartan formula to $\tilde{Q}^{r+1}\left(Q^{r}\left(e_{0}\right) *[1] *\left(\chi e_{0}^{2}\right)\right)$.

LeMma 5.1.11. In $H_{*}(S G(\mathbf{Z} / 2), \mathbf{Z} / 2)$ with $r>\alpha$

$$
\tilde{Q}^{r+\alpha+1}\left(Q^{r}\left(e_{\alpha}\right) *[1] * \chi e_{0}^{2}\right)=Q^{r+\alpha+1} Q^{r}\left(e_{\alpha}\right) *[1] * \chi e_{0}^{3}
$$

modulo higher weight.

Proof. Using May's formula, [6], $\tilde{Q}^{r}\left(e_{\alpha}\right)$ may be written as a sum $\sum c_{s}\left(Q^{r+s}(1) \circ e_{\alpha-s}\right)$ where $c_{s}$ is some binomial coefficient. But

$$
\begin{aligned}
\tilde{Q}^{r+\alpha+1}\left(Q^{r+s}(1) \circ e_{\alpha-s}\right)= & \tilde{Q}^{r+s+1} Q^{r+s}(1) \circ \tilde{Q}^{\alpha-s}\left(e_{\alpha-s}\right) \\
& +\tilde{Q}^{r+s} Q^{r+s}(1) \circ \tilde{Q}^{\alpha-s+1}\left(e_{\alpha-s}\right) .
\end{aligned}
$$

As $n=1, \tilde{Q}^{\alpha-s} e_{\alpha-s}=e_{\alpha-s} * e_{\alpha-s}$; thus as in 5.1.10 the above sum is zero. Applying the mixed Cartan formula to $\tilde{Q}^{r+\alpha+1}\left(Q^{r} e_{\alpha} *[1] * \chi e_{0}^{2}\right)$ gives the result. 
LEMMA 5.1.12. Let $n>1$, then in $H_{*}\left(S G\left(\mathbf{Z} / 2^{n}\right), \mathbf{Z} / 2\right)$ with $r>\alpha$,

$$
\begin{aligned}
& \tilde{Q}^{r+\alpha+1}\left(Q^{r}\left(e_{\alpha}\right) *[1] * \chi e_{0}^{2}\right) \\
& \quad=Q^{r+\alpha+1} Q^{r}\left(e_{\alpha}\right) *[1] * \chi e_{0}^{3}+\sum Q^{S_{1}} Q^{S_{2}}\left(e_{\beta}\right) *[1] * \chi e_{0}^{3}
\end{aligned}
$$

modulo higher weight where the sum runs over certain $\left(S_{1}, S_{2}, \beta\right) \neq(r+\alpha$ $+1, r, \alpha)$.

Proof. As $Q^{r}\left(e_{\alpha}\right)=\sum_{s} Q^{r+s}(1) \circ e_{\alpha-s}$ for certain $s$ we have

$$
\begin{aligned}
& \tilde{Q}^{r+\alpha+1}\left(Q^{r}\left(e_{\alpha}\right)\right) \\
& \quad=\sum_{s}\left(\tilde{Q}^{r+s+1} Q^{r+s}(1) \circ \tilde{Q}^{\alpha-s}\left(e_{\alpha-s}\right)\right)+\tilde{Q}^{r+s} Q^{r+s}(1) \circ \tilde{Q}^{\alpha-s+1}\left(e_{\alpha-s}\right) \\
& \quad=\sum_{s} \tilde{Q}^{r+s+1} Q^{r+s}(1) \circ \tilde{Q}^{\alpha-s}\left(e_{\alpha-s}\right)
\end{aligned}
$$

as before. Again, $\tilde{Q}^{r+s+1} Q^{r+s}(1)=Q^{2 r+2 s+1}(1)$ modulo higher weight and thus the terms of lowest weight in the sum are $\Sigma_{s} Q^{2 r+2 s+1}(1) \circ \tilde{Q}^{\alpha-s}\left(e_{\alpha-s}\right)$. Unfortunately, $\tilde{Q}^{\alpha-s}\left(e_{\alpha-s}\right)$ is not a loop sum square (as $n>1$ ) but 5.1.3 and 1.3.3 imply the elements of lowest weight in the sum above are $\sum Q^{2 r+2 s+1+q}\left(Q^{\alpha-s+t+k-q+w}\left(e_{\alpha-s-t-k-w}\right)\right)$ where the sum runs over certain $s, q, t, k$, and $w$. But the partial sum of the terms where $s=t=k=$ $w=0$ is equal to $Q^{2 r+1}(1) \circ\left(e_{\alpha} * e_{\alpha}\right)$ which is zero as before. Once again the mixed Cartan formula gives the desired result.

LEMMA 5.1.13. In $H_{*}\left(S G\left(\mathbf{Z} / p^{n}\right), \mathbf{Z} / p\right)$ for all $p$ and all $n$ if $l(I) \geq 2$, and $e(I)>\alpha$ then $\tilde{Q}_{1}\left(Q^{I}\left(e_{\alpha}\right) *[1] * \chi e_{p}^{p^{I(I)}}\right) \equiv Q_{1} Q^{I}\left(e_{\alpha}\right) *[1] * \chi\left(e_{0}^{p^{l(I)+1}}\right)$ modulo higher weight.

Proof. A slight generalization of $[5,8.3 .1]$ or $[6,1.7]$ shows $Q^{I}\left(e_{\alpha}\right)=$ $\sum Q^{i_{1}}(1) \circ \cdots \circ Q^{i_{k}}(1) \circ e_{\beta}$, where $\beta>0$ if and only if $\alpha>0$ and $l(I)=k$. The rest of the proof follows exactly as in $[5,8.3$.iii] or $[6,5.7]$.

Proof of 5.1.9. 5.1.13 proves 5.1.9 if $l(I) \geq 2$. Minor modification of the arguments of 5.1.10 and 5.1.11 for $p$ odd as in [6] handle $l(I)=1$. We leave the details to the reader.

LEMMA 5.1.14. Let $n=1, p=2$. Then

$$
\tilde{Q}_{1}\left(J_{\mathbf{Z} / 2}\left(e_{r}\right) * J_{\mathbf{Z} / 2}\left(e_{r}\right) *[-1]\right)=0 \text {. }
$$


Proof. Apply the mixed Cartan formula to obtain

$$
\begin{aligned}
\sum_{\substack{i+j=0 \\
s_{0}+s_{1}+s_{2}=1}} \tilde{Q}_{s_{0}}\left(J_{\mathbf{Z} / 2}\left(e_{i}\right)\right) * J_{\mathbf{Z} / 2}\left(e_{i}\right) * \tilde{Q}_{s_{1}}([-1]) \\
* Q_{s_{2}}\left(J_{\mathbf{Z} / 2}\left(e_{j}\right) * J_{\mathbf{Z} / 2}\left(e_{j}\right) \circ[-1]\right)
\end{aligned}
$$

by symmetry. But $J_{\mathrm{Z} / 2}\left(e_{j}\right) * J_{\mathrm{Z} / 2}\left(e_{j}\right) \circ[-1]=0$.

LemMA 5.1.15. Let $n>1, p=2$. Then

$$
\tilde{Q}_{1}\left(J_{n}\left(e_{2 r+1}\right) * J_{n}\left(e_{2 r+1}\right) *[-1]\right)=0 .
$$

Proof. $[13,7.10]$.

5.2. We conclude this chapter by considering the geometric twist action map defined in Chapter IV. From Chapter IV we know the following diagram commutes:

$$
\begin{array}{ccc}
C \times K^{H} & \stackrel{\bar{\lambda}}{\rightarrow} & K^{H} \\
\downarrow \text { id } \times \Phi & & \downarrow \Phi \\
C \times F(H) & \stackrel{\lambda^{\prime \prime}}{\rightarrow} & F(H) \\
\uparrow \operatorname{id} \times \rho & & \uparrow \rho \\
C \times S G(H) & \stackrel{\lambda^{\prime}=\Omega \psi_{G}}{\rightarrow} & S G(H)
\end{array}
$$

Classifying this diagram we obtain

$$
\begin{array}{ccc}
B C \times B K^{H} & \rightarrow & B K^{H} \\
\downarrow \text { id } \times B \Phi & & \downarrow B \Phi \\
B C \times B F(H) & \rightarrow & B F(H) \\
\uparrow \operatorname{id} \times \rho & & \uparrow \text { id } \times \rho \\
B C \times B S G(H) & \stackrel{\psi_{G}}{\rightarrow} & B S G(H)
\end{array}
$$

Now let $H=\mathbf{Z} / 2$. As 2.3 .1 identifies the $\mathfrak{L}^{H}$ structure with the composition product structure, diagram 5.2.2 for $H=\mathbf{Z} / 2$ is compatible with diagram 1.4.3. Thus we may loop 5.2.2 for $H=\mathbf{Z} / 2$ to obtain a diagram compatible with 1.4.4. Thus [12.2.6] implies 1.4.6. 1.4.7 follows from the properties of the $\mathbf{Z} / 2$ equivariant $J$ homomorphism once we prove 1.4.5. Finally, results of Thomason and Wilson [32], implies the homology suspension commutes with the action map which in turn yields 1.4.8. 
Proof of 1.4.5. This result is standard. It follows from the following well-known lemma which may be found in [31].

LEMMA 5.2.3.

(a) $H^{*}(B O, \mathbf{Z} / 2) \cong P\left[w_{i} \mid i \geq 1\right]$ where $w_{i}$ are the Stiefel-Whitney classes.

(b) $H^{*}(B O, \mathbf{Z} / 2)$ has an additive basis consisting of $s_{w}(\sigma)$ as $w$ runs over partitions $w=\left(i_{1}, \ldots, i_{r}\right)$ with coproduct $\psi\left(s_{w}(\sigma)\right)=\sum_{w=w_{1} \amalg w_{2}} s_{w_{1}}(\sigma) \otimes$ $s_{w_{2}}(\sigma)$.

(c) $s_{i}(\sigma)$ is dual to $z_{i}$ defined in $\S 1.4$.

(d) The Pontrjagin product $z_{i_{1}} \circ \cdots \circ z_{i_{r}}$ in $H_{*}(B O, \mathbf{Z} / 2)$ is dual to $s_{\left(i_{1}, \ldots, i_{r}\right)}(\sigma)$ in the $\left\{s_{w}(\sigma)\right\}$ basis.

LEMMA 5.2.4. The map

$$
S^{1} \times \mathbf{R} P^{\infty} \rightarrow S^{1} \wedge\left(\mathbf{R} P_{+}^{\infty}\right) \stackrel{S^{1} \wedge i}{\hookrightarrow} S^{1} \wedge 0_{+} \hookrightarrow B O
$$

classifies the virtual bundle $(\hat{L}-1) \otimes L$ over $S^{1} \times \mathbf{R} P^{\infty}=\mathbf{R} P^{1} \times \mathbf{R} P^{\infty}$. Here $\hat{L} \rightarrow \mathbf{R} P^{1}, L \rightarrow \mathbf{R} P^{\infty}$ are the canonical nontrivial line bundles.

Proof. The virtual bundle $E^{n}-\bar{n}$ over $S^{1} \wedge O(n)_{+}$induced by the inclusion $S^{1} \wedge O(n)_{+} \hookrightarrow B O$ is defined by using the clutching function $O(n) \stackrel{\text { id }}{\rightarrow} O(n)$ to define the bundle $E^{n} \rightarrow S^{1} \wedge O(n)_{+}$. Now $i_{n}: \mathbf{R} P^{n-1} \hookrightarrow$ $O(n)$ is given by sending a line $l$ in $\mathbf{R}^{n}$ to the map determined by

$$
\left\{\begin{array}{ll}
\vec{x} \rightarrow \vec{x} & \text { for } x \in l \\
\vec{x} \rightarrow-\vec{x} & \text { for } x \in l^{\perp}
\end{array}\right\}
$$

Hence the bundle $\left.E^{n}\right|_{S^{1} \wedge \mathbf{R} P_{+}^{n-1}}$ is defined by the clutching map $i_{n}$.

Thus the pull-back of $E^{n}$ to $S^{1} \times \mathbf{R} P^{n-1}$ is described as follows. First clutch the complementary bundle $Q, \quad\left\{l \in \mathbf{R} P^{n-1} \rightarrow l^{\perp} \subset \mathbf{R}^{n}\right\}$, by the identity map and clutch the subplane bundle $L,\left\{l \in \mathbf{R} P^{n-1} \rightarrow l \subset \mathbf{R}^{n}\right\}$, by the antipodal map. Then the pull-back of $E^{n}$ to $S^{1} \times \mathbf{R} P^{n-1}$ is $1 \otimes Q \oplus \hat{L} \oplus L$ where $\hat{L}$ is the "Möbius band" bundle over $S^{1}$. Hence the pull-back of the universal bundle $\xi$ over $B O$ of the composition $S^{1} \times$ $\mathbf{R} P^{n-1} \rightarrow S^{1} \wedge \mathbf{R} P^{n-1} \rightarrow S^{1} \wedge O_{(n)_{+}} \rightarrow B O_{(n)} \rightarrow B O$ is $1 \otimes Q+\hat{L} \otimes L-$ $1 \otimes \bar{n}$. But over $\mathbf{R} P^{n-1}$ we have the equation $\bar{n}=Q \otimes L$. Thus

$$
\begin{gathered}
1 \otimes Q+\hat{L} \otimes L-1 \otimes \bar{n}=1 \otimes(Q-\bar{n})+\hat{L} \otimes L \\
=\hat{L} \otimes L-1 \otimes L=(\hat{L}-1) \otimes L .
\end{gathered}
$$


We may now prove 1.4.5.c.

LEMMA 5.2.5.

$$
y_{s}=\sum_{w=\left(i_{1}, \ldots, i_{r}\right)} N(w) z_{i_{1}} \circ \cdots \circ z_{i_{r}}, \quad \sum_{j=1}^{n} i_{j}=s .
$$

Proof. Recall for $w=\left(i_{1}, \ldots, i_{r}\right)$ we may write $s_{w}(\sigma) \in H^{|w|}(B O, \mathbf{Z} / 2)$ as $s_{w}(\sigma)=N(w) w_{|w|}+$ decomposables. Using 4.1.6 we calculate as follows

$$
\begin{aligned}
\left(j^{*} \circ S^{1} \wedge i_{s}^{*}\right) w_{*}(\xi) & =w_{*}(\hat{L} \otimes L-1 \otimes L) \\
& =\frac{1+w_{1}(L)+w_{1}(\hat{L})}{1+w_{1}(L)}=1+w_{1}(\hat{L}) \otimes \frac{1}{1+w_{1}(L)}
\end{aligned}
$$

where $w_{*}$ is the total Stiefel-Whitney class. That is, for $i \geq 1, w_{i}(\xi) \rightarrow$ $w_{1}(L) \otimes w_{1}(L)^{i-1}$ but $j^{*} \circ\left(S^{1} \wedge i_{s}\right)^{*}$ sends decomposables to zero. A calculation shows $j^{*} \circ\left(S^{1} \wedge i_{s}\right){ }^{*} s_{W}(\sigma)=N(w)$. The result follows from 5.2.3.

LEMMA 5.2.6. The following diagram commutes.

$$
\begin{array}{ccc}
\mathbf{R} P_{+}^{\infty} \wedge B O & \stackrel{\tilde{L} \otimes \xi}{\rightarrow} & B O \\
\mathbf{R} P_{+}^{\infty} \wedge\left(S^{1} \wedge\left(\mathbf{R} P_{+}^{\infty}\right)\right) & \\
\| & \uparrow j\left(S^{1} \wedge i\right) \\
S^{1} \wedge\left(\mathbf{R} P_{+}^{\infty}\right) \wedge\left(\mathbf{R} P_{+}^{\infty}\right) & \\
\| & \\
S^{1} \wedge\left(\mathbf{R} P^{\infty} \times \mathbf{R} P^{\infty}\right)_{+} & \stackrel{S^{1} \wedge(L \otimes L)}{\rightarrow} & S^{1} \wedge \mathbf{R} P_{+}^{\infty}
\end{array}
$$

Proof. Obvious.

Corollary 5.2.7.

$$
\psi_{0_{*}}\left(e_{p},\left(j \circ\left(S^{1} \wedge i\right)\right)_{*}\left(S^{1} \wedge e_{q}\right)\right)=\left(j \circ\left(S^{1} \wedge i\right)\right)_{*}\left(S^{1} \wedge(p, q) e_{p+q}\right) .
$$

Proof. Follows from 5.2.6 as $\mathbf{R} P^{\infty} \times \mathbf{R} P_{\stackrel{\infty}{\check{L} \otimes L}}^{\rightarrow} \mathbf{R} P^{\infty}$ sends $e_{p} \otimes e_{q}$ to $(p, q) e_{p+q} \cdot 5.2 .7$ implies 1.4.5(b). 
The commutative diagram

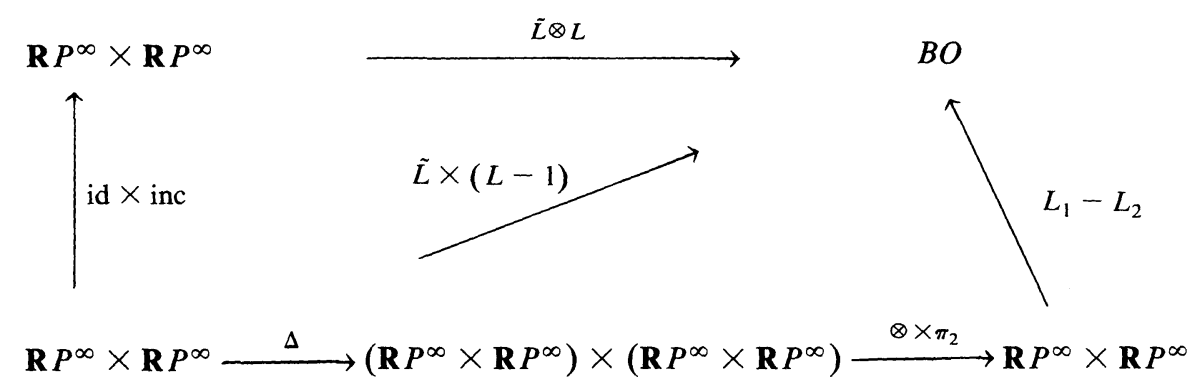

yields 1.4.5a since in homology

$$
\begin{aligned}
& e_{p} \otimes z_{r} \\
& \uparrow \\
& e_{p} \otimes e_{r} \rightarrow \sum_{\substack{p=p_{1}+p_{2} \\
r=r_{1}+r_{2}}}\left(e_{p_{1}} \otimes e_{r_{1}}\right) \otimes\left(e_{p_{2}} \otimes e_{r_{2}}\right) \rightarrow \sum_{r=r_{1}+r_{2}}\left(p, r_{1}\right) e_{p+r_{1}} \otimes e_{r_{2}}
\end{aligned}
$$

as the right-hand vertical map is zero unless $p_{2}=0$.

Before we prove $1.4 .5 \mathrm{~d}$ we note that the equation $L \otimes\left(\xi_{1} \oplus \xi_{2}\right)=$ $\left(L \otimes \xi_{1}\right) \oplus\left(L \otimes \xi_{2}\right)$ implies

LEMMA 5.2.9.

(a) $\psi_{0_{*}}\left(e_{n}, x \circ y\right)=\Sigma_{i+j=n} \psi_{0_{*}}\left(e_{i}, x\right) \circ \psi_{0_{*}}\left(e_{j}, y\right)$.

(b) $\Omega \psi_{0_{*}}\left(e_{n}, x \circ y\right)=\sum_{i+j=n} \Omega \psi_{0_{*}}\left(e_{i}, x\right) \circ \Omega \psi_{0_{*}}\left(e_{j}, y\right)$.

Proof. The commutative diagram.

$$
\begin{gathered}
\mathbf{R} P^{\infty} \wedge[B O \times B O]_{+} \stackrel{\mathrm{id} \times(\oplus)}{\rightarrow} \mathbf{R} P^{\infty} \wedge B O_{+} \\
\downarrow \Delta \\
\left(\mathbf{R} P^{\infty} \wedge B O_{+}\right) \times\left(\mathbf{R} P^{\infty} \wedge B O_{+}\right) \stackrel{\psi_{0} \times \psi_{0}}{\rightarrow} B O \times B O \stackrel{\oplus}{\rightarrow} B O
\end{gathered}
$$

represents $L \otimes\left(\xi_{1} \oplus \xi_{2}\right)=\left(L \otimes \xi_{1}\right) \oplus\left(L \otimes \xi_{2}\right)$. This diagram loops down to give

$$
\begin{aligned}
& \mathbf{R} P^{\infty} \times 0 \times 0 \stackrel{\text { id } \times \text { product }}{\rightarrow} \mathbf{R} P^{\infty} \times 0 \\
& \downarrow \Delta \\
& \mathbf{R} P^{\infty} \times 0 \times \mathbf{R} P^{\infty} \times 0 \stackrel{\Omega \psi_{0} \times \Omega \psi_{0}}{\rightarrow} 0 \times 0 \stackrel{\text { product }}{\rightarrow} 0
\end{aligned}
$$

The lemma follows. 
Finally 1.4.5.d follows from the next lemma.

LEMMA 5.2.10. The following diagram commutes up to homotopy.

$$
\begin{array}{ccc}
\mathbf{R} P^{\infty} \times \mathbf{R} P^{\infty} & \stackrel{\tilde{L} \otimes L}{\rightarrow} & \mathbf{R} P^{\infty} \\
\downarrow \text { id } \times i & & \downarrow i \\
\mathbf{R} P^{\infty} \times 0 & \rightarrow & 0
\end{array}
$$
grams

Proof. The diagram in 5.2.6 yields the following commutative dia-

$$
\begin{aligned}
& \mathbf{R} P_{+}^{\infty} \wedge \mathbf{R} P_{+}^{\infty} \quad \stackrel{\otimes}{\rightarrow} \mathbf{R} P_{+}^{\infty} \\
& \begin{array}{ccccc}
\stackrel{\operatorname{id} \times i \swarrow}{ } & \downarrow \text { id } \wedge i & & \downarrow i & \searrow i \\
\mathbf{R} P_{+}^{\infty} \wedge 0_{+} & \mathbf{R} P_{+}^{\infty} \wedge \Omega\left(S^{1} \wedge \mathbf{R} P_{+}^{\infty}\right) & \stackrel{\otimes}{\rightarrow} & \Omega\left(S^{1} \wedge \mathbf{R} P_{+}^{\infty}\right) & 0_{+}
\end{array} \\
& \downarrow \quad \downarrow \Omega\left(S^{1} \wedge i\right) \\
& \searrow \sim \quad \mathbf{R} P_{+}^{\infty} \wedge \Omega\left(S^{1} \wedge 0_{+}\right) \quad \Omega\left(S^{1} \wedge 0_{+}\right) \quad \lambda \sim \\
& \downarrow \quad \downarrow \Omega_{j} \\
& \mathbf{R} P_{+}^{\infty} \wedge \Omega B O \quad \stackrel{\Omega \psi_{0}}{\rightarrow} \Omega B O \\
& \begin{array}{ccc}
\uparrow & \uparrow \| l \\
\mathbf{R} P_{+}^{\infty} \wedge 0 & \Omega \psi_{0} & 0
\end{array}
\end{aligned}
$$

and hence

$$
\begin{aligned}
& \mathbf{R} P_{+}^{\infty} \wedge \mathbf{R} P_{+}^{\infty} \stackrel{\otimes}{\rightarrow} \quad \mathbf{R} P_{+}^{\infty} \\
& \measuredangle \text { id } \times i \\
& \mathbf{R} P_{+}^{\infty} \wedge 0_{+} \\
& \searrow \sim \quad \prec \sim
\end{aligned}
$$

which gives the lemma up to homotopy.

\section{APPENDIX}

We will prove Theorems 3.1.3 and 3.1.6. Actually we prove these theorems using a map $\gamma_{B S}^{\prime}: G(H) \rightarrow Q\left(B H^{+}\right)$which is a variant of the Becker-Schultz equivalence $\gamma_{B S} \cdot \gamma_{B S}^{\prime}$ was used in [13]. It is a homotopy equivalence that respects composition product and is natural with respect to transfers. For details of the comparison between $\gamma_{B S}$ and $\gamma_{B S}^{\prime}$ we refer the reader to [13]. 
We begin by recalling Segal's equivalence $\gamma_{S}$.

Given a map $M \stackrel{\varphi}{\rightarrow} \operatorname{Map}_{H}(t(n V \oplus m R))$ of a smooth compact, closed manifold $M$, Segal produces a map $M \rightarrow Q\left(S^{0}\right) \times Q\left(B H^{+}\right)$. Given $\varphi$ this is done by first deforming $\varphi$ so that the induced $H$ equivariant map $H \times t(n V \oplus m R) \stackrel{\phi}{\rightarrow} t(n V \oplus m R)$ is transverse to $\overrightarrow{0} \in(n V \oplus m R) \subset$ $t(n V \oplus m R)$. Then $\phi^{-1}(0) \subset M \times(n V \oplus m R)$ is an $H$-equivariant submanifold of the $H$-equivariant manifold $M \times(n V \oplus m R)$ with an $H$ equivariant trivialization of its normal bundle with $(n V \oplus m R) \times M$. As such the isotopy groups of $\phi^{-1}(0)$ are constant and $\phi^{-1}(0)$ may be canonically expressed as the union of disjoint closed $H$-equivariant submanifolds $A=\phi^{-1}(0) \cap(0 \times m R)$ in the fixed part and $B=\phi^{-1}(0) \cap$ $((n V-\{0\}) \times m R)$ in the free part of $n V \oplus m R$ since the isotopy groups of $n V \oplus m R$ are id and $H$ as $H$ acts freely in $S(V)$.

$$
\phi^{-1}(0)=A \amalg B .
$$

Furthermore, the equivariant trivialization of the normal bundle of $\phi^{-1}(0)$ in $n V \oplus m R$, yield trivializations

$$
\begin{gathered}
\nu(A \hookrightarrow(0 \times m R) \times M) \stackrel{\alpha}{\approx} R^{m} \times A, \\
\nu(B / H \hookrightarrow((m V-\{0\}) / H \times m R) \times M) \stackrel{\beta}{\approx} R^{m+n \operatorname{dim} V} \times B
\end{gathered}
$$

of the normal bundle of $A$ (respectively $B$ ) in the fixed point set (respectively the quotient of the free $H$ action).

The pair $(A \hookrightarrow(0 \times m R) \times M, \alpha)$ describes a stable map $S^{N} \wedge \mathrm{M} \rightarrow$ $S^{N}$; that is, a map $M \rightarrow Q\left(S^{0}\right)$; the pair $(B \hookrightarrow((n V-\{0\}) / H \times m R) \times$ $M, \beta)$ describe a stable map $S^{N} \wedge M \rightarrow S^{N} \wedge((n V-\{0\} / H) \times m R)^{+}$; that is, a map $M \rightarrow Q\left(B H^{+}\right)$where we use the classifying map $(n V-(0) / H) \times m R \rightarrow B H$ of the free action on $(n V \oplus m R-$ $(0 \times m R))$. Together these give the desired map $M \rightarrow Q\left(S^{0}\right) \times Q\left(B H^{+}\right)$.

We remark that when $m=0 \quad \gamma_{S}$ clearly maps $F(H)$ into [1] $\times$ $Q\left(B H^{+}\right)$. We next describe $\gamma_{B S}^{\prime}$.

Given a map $M \stackrel{\psi}{\rightarrow} \operatorname{Map}_{H}(S(n V))$ of a smooth compact closed manifold $M$, we associate a map $M \rightarrow Q\left(B H^{+}\right)$as follows:

Deform $\psi$ so that the induced $H$-equivariant map $\hat{\psi}: M \times S(n V) \rightarrow$ $M \times S(n V)$ has

$$
\left(\hat{\psi}, \mathrm{pr}_{2}\right): M \times S(N V) \rightarrow S(N V) \times S(N V)
$$


transverse to the diagonal $\Delta: S(N V) \rightarrow S(N V) \times S(N V)$. Then the $H$ equivariant submanifold $P=\left(\hat{\psi}, \mathrm{pr}_{2}\right)^{-1}(\mathrm{im} \Delta) \subset M \times S(N V) \subset M \times$ $N V$ has normal bundle $H$-equivariantly trivialized by the bundle maps covering

$$
\begin{aligned}
\nu(P \hookrightarrow M \times N V) \cong \nu(P \hookrightarrow M \times S(N V)) \oplus \bar{R} \\
\downarrow \\
\nu(S(N V) \stackrel{\Delta}{\hookrightarrow} S(N V) \times S(N V)) \oplus \bar{R} \\
\downarrow \\
\left.\downarrow \text { via( } \mathrm{pr}_{2} \times \text { id }\right) \\
N \bar{V}=T_{*}(S(N V)) \oplus \bar{R}
\end{aligned}
$$

(since the normal bundle of $S(N V) \hookrightarrow S(N V) * S(N V)$ is isomorphic to the tangent bundle $T_{*} S(N V)$ of $\left.S(N V)\right)$.

This $H$-equivariant trivialization yields a trivialization of the normal bundle $\nu(P / H \hookrightarrow M \times(N V-\{0\} / H))$ and thus a stable map $S^{N} \wedge M$ $\rightarrow S^{N} \wedge(N V-\{0\} / H)^{+}$. This describes a map $M \rightarrow Q\left(B H^{+}\right)$where we use the classifying map $N V-\{0\} / H \rightarrow B H$ of the free action of $H$ on $N V-\{0\}$.

If $W$ is an even-finite dimensional subspace of $n V$ we define a map

$$
\delta: \operatorname{Map}_{H}(S(W)) \rightarrow \operatorname{Map}_{H}(t(W))
$$

by the formulae:

$$
\delta(g)(\infty)=\infty
$$

and

$$
\delta(g)(\lambda \hat{x})= \begin{cases}-\lambda \hat{x} & \text { if } 0 \leq \lambda \leq 1 / 2 \\ -1 / 2 \hat{x}+(\lambda-1 / 2) g(\hat{x}) & \text { if } \lambda \geq 1 / 2\end{cases}
$$

REMARKS.

(a) $g: S(W) \rightarrow S(W)$ and $\|\hat{x}\|=1$.

(b) $\delta(g)$ restricted to the disk of radius $1 / 2$ is just the antipodal map.

(c) $\delta(g)$ extends continuously to $t W$ as $\|\delta(g)(\lambda \hat{x})-\lambda g(\hat{x})\|=$ $\left\|-\frac{1}{2}(\hat{x}+g(\hat{x}))\right\| \leq 1$.

Thus we may define a homotopy

$$
\delta_{t}(g)(\lambda \hat{x})=(1-t) \lambda g(\hat{x})+t \delta(g)(\lambda \hat{x})
$$

between $\mathcal{S}_{0}(g)=\rho g$ and $\delta_{1}(g)=\delta(g)$. 
An easy computation shows

$$
\delta(g)^{-1}(0)=\{0\} \coprod\{\hat{x} \mid g(\hat{x})=\hat{x}\} .
$$

Then given $\hat{\psi}, P$ and the trivialization above (recall the description of $\left.\gamma_{B S}^{\prime}\right) \phi(\delta(\hat{\psi})): M \rightarrow \operatorname{Map}_{H}(t(W))$ is given by the adjoint of $(m, \lambda \hat{x}) \rightarrow$ $(m, \mathcal{S}(\hat{\psi}(m))(\lambda \hat{x}))$. By (A5) we conclude

$$
\phi(\delta \hat{\psi})^{-1}(M \times \overrightarrow{0})=M \times 0 \coprod P .
$$

The framings are compatible and thus induce the following commutative diagram

$$
\begin{aligned}
& \operatorname{Map}(S(W)) \quad \stackrel{\rho}{\rightarrow} \quad \operatorname{Map}(t(W)) \\
& \downarrow \gamma_{B S} \quad \downarrow \gamma_{S} \\
& Q\left(B H^{+}\right) \rightarrow[1] \times Q\left(B H^{+}\right) \quad \rightarrow \quad Q S^{0} \times Q\left(B H^{+}\right)
\end{aligned}
$$

As $W$ is even dimensional (A7) is compatible with limits (see [13]). This proves 3.1.3.

To prove 3.1.6 we first recall from [9] the identification of $K^{H}$. As pointed out by May

$$
\left(\mathscr{K}(j) / \Sigma_{j}\right)^{H} \simeq \coprod_{\varphi} \mathscr{K}(j)^{\varphi} / \Sigma_{\varphi}
$$

where

(a) $\varphi$ runs through conjugacy classes of homomorphisms $\varphi: H \rightarrow \Sigma_{j}$.

(b) $\mathscr{K}(j)^{\varphi}$ is the subset of $\mathscr{K}(j)$ consisting of all points $k$ such that $h k=k \varphi(h)$ for all $h$ in $H$.

(c) $\Sigma_{\varphi}$ is the subgroup of $\Sigma_{j}$ consisting of all elements $\sigma$ such that $\sigma \varphi(h)=\varphi(h) \sigma$ for every $h$ in $H$.

(d) As $\mathscr{K}(j)=\lim _{W} \mathscr{K}(j, W)$ is the limit of semi-free $H$ actions on finite dimensional subspaces of $(V \oplus \mathbf{R})_{H}, \mathscr{K}(j)^{\varphi}$ is empty unless $\varphi$ corresponds to a semi-free action. If $\varphi$ does correspond to a semi-free action then $\mathscr{K}(j)^{\varphi}$ is contractible and $\mathscr{K}(j)^{\varphi} / \Sigma_{\varphi}$ is a $K(\pi, 1)$.

Hence we obtain the decomposition

$$
K^{H} \cong \coprod_{j} \coprod_{\varphi_{J}}\left(\mathcal{K}(j)^{\varphi_{J}} / \Sigma_{\varphi_{J}}\right) \cong \coprod_{n} B \Sigma_{n} \times \coprod_{m} B\left(\Sigma_{m} \int H\right)
$$


where the $\left(\mathscr{K}^{H}, \mathfrak{L}^{H}\right)$ operad action on $K^{H}$ is described on $\amalg_{n} B \Sigma_{n} \times$ $\amalg_{m} B\left(\Sigma_{m} \int H\right)$ and hence on a group completion $Q\left(S^{0}\right) \times Q\left(B H^{+}\right)$by the maps of 2.2 .4 restricted to the $K(\pi, 1)$ level.

As $K^{H} \stackrel{\phi}{\rightarrow} F(H) \stackrel{\gamma_{S}}{\rightarrow} Q\left(S^{0}\right) \times Q\left(B H^{+}\right)$is clearly a $K^{H}$ map to prove 3.1.6 it suffices to check

(a) The composite

$$
S^{0} \wedge B H^{+} \hookrightarrow K^{H} \stackrel{\phi}{\rightarrow} F(H) \stackrel{\gamma_{S}}{\rightarrow} Q\left(S^{0}\right) \times Q\left(B H^{+}\right)
$$

is homotopic to the standard inclusion.

(b) More generally, the composites

$$
B \Sigma_{\alpha} \times B\left(\Sigma_{\beta} \int H\right) \hookrightarrow K^{H} \stackrel{\phi}{\rightarrow} F(H) \stackrel{\gamma_{s}}{\rightarrow} Q\left(S^{0}\right) \times Q\left(B H^{+}\right)
$$

are homotopic to the standard inclusion.

(a) is proved by starting with an approximation to $B H$, say a compact smooth manifold $M$ and a map $M \stackrel{\tau}{\rightarrow} B H$ which is $N$-connected. This map may be replaced by a smooth map

$$
M \stackrel{f}{\rightarrow}(n V-\{0\}) / H \times m R
$$

for $n$ large. (Note that the right-hand side is a smooth manifold.) We may regard $f$ as a map $M \rightarrow$ configurations of one free $H$-orbit in ( $n V-\{0\})$ $\times m R\}$. That is, for $x \in M$, we have $f(x)=\left(x_{1}, x_{2}, \ldots, x_{|H|}\right)$ where $x_{1}, \ldots, x_{|H|}$ is a free $H$-orbit of $n V \oplus m R$. The map $\phi \circ f: M \rightarrow \operatorname{Map}_{H}(t(n V$ $\oplus m R)$ ) is then defined by

$$
f(x)(y)= \begin{cases}\frac{y-x_{i}}{\varepsilon^{2}-\left|y-x_{i}\right|^{2}} & \text { if } y \in\left(\varepsilon \text {-ball about } x_{i} \text { for some } i\right), \\ \infty & \text { otherwise, }\end{cases}
$$

where $f(x)=\operatorname{orbit}\left(x_{1}, x_{2}, \ldots, x_{\mid H}\right)$, and $\varepsilon=1 / 2$ (minimum distance between the $x_{i}$ 's). (We have chosen an $H$-invariant metric on $n V \oplus m R$.)

Now the composite $\gamma_{S} \circ \phi \circ f: M \rightarrow Q\left(S^{0}\right) \times Q(B H)$ is easy to describe since $\phi \circ f(x)$ is already transverse to $0 \in n V \oplus m R$. By our prescription above, $\phi \circ f$ induces a map

$$
\begin{aligned}
\Psi: M \times t(n V \oplus m R) & \rightarrow t(n V \oplus m R), \\
(x, y) & \rightarrow(\phi \circ f)(x)(y)
\end{aligned}
$$


with

$$
\begin{aligned}
\Psi^{-1}(0) & =\left\{(x, z) \mid z \text { is in the free } H \text {-set } f(x)=\left(x_{1}, x_{2}, \ldots, x_{|H|}\right)\right\} \\
& =\pi^{-1}(\text { graph of } M \stackrel{f}{\rightarrow}(n V-\{0\} / H) \times m R)
\end{aligned}
$$

where $\pi:(M \times(n V-\{0\}) \times m R) \rightarrow M \times(n V-\{0\} / H) \times m R$ is the projection $\mathrm{pr}_{2}$ which induces the isomorphism

$$
\nu\left(\Psi^{-1}(0) \hookrightarrow M \times(n V-\{0\}) \times m R\right) \stackrel{\approx}{\rightarrow}(n V \oplus m R) \times \Psi^{-1}(0) .
$$

Hence, $\gamma_{S} \circ \phi \circ f$ is described by $M \rightarrow[0] \in Q\left(S^{0}\right)$ and $M \rightarrow Q\left(B H^{+}\right)$. The latter is given by $M_{\alpha}=\{$ graph of $M \stackrel{f}{\rightarrow}(n V-\{0\} / H) \times m R\}$ with the isomorphism

$$
\begin{aligned}
\nu(M & \hookrightarrow M \times(n V-\{0\} / H) \times m R) \\
& \simeq\left(\mathrm{pr}_{2}\right) *\left(T_{*}((n V-\{0\} / H) \times m R)\right)
\end{aligned}
$$

coming from the projection $\mathrm{pr}_{2}$ which defines a map

$$
M \rightarrow Q((n V-\{0\} / H) \times m R) \rightarrow Q\left(B H^{+}\right)
$$

by classifying the free $H$-action on $(n V-(0) / H) \times m R$. That is, this second map $M \rightarrow Q\left(B H^{+}\right)$is merely $M \stackrel{f}{\rightarrow} B H \stackrel{i}{\rightarrow} Q\left(B H^{+}\right)$where $i$ is the standard inclusion. This proves (a).

The proof of (b) is similar. Briefly, given $g: M \rightarrow B \Sigma_{\alpha} \times B\left(\Sigma_{\beta} \int H\right)$ which is $N$-connected, we replace $g$ by a smooth map

$$
f: M \rightarrow\left\{\begin{array}{l}
\text { configurations of } \\
\text { points in } 0 \times m R
\end{array}\right\} \times\left\{\begin{array}{l}
\text { configurations of free } H \text {-orbits } \\
\text { in }(n V-\{0\}) \times m R
\end{array}\right\}
$$

and then proceed as before to get property (b).

This concludes the proof of Theorem 3.1.6.

\section{REFERENCES}

[1] M. G. Barrett, V. K. A. Gugenheim, and J. C. Moore, On semisimple fibre-bundles, Amer. J. Math., 81 (1959), 639-657.

[2] J. C. Becker and R. E. Schultz, Equivariant function spaces and stable homotopy theory, Comm. Hel. Math., 49 (1974), 1-34.

[3] Equivariant function spaces and stable homotopy theory II, Indiana U. Math. J., 25 (1976), 481-492. 
[4] J. M. Boardman and R. M. Vogt, Homotopy invariant algebraic structures on topological spaces, L.N.M. vol. 347, Springer-Verlag, 1973.

[5] G. Brumfiel, I. Madsen, and R. J. Milgram, PL charactertistic classes and cobordism, Ann. of Math., 97 (1973), 82-159.

[6] F. R. Cohen, T. J. Lada, and J. P. May, The homotopy of iterated loop spaces, L.N.M. vol. 533, Springer-Verlag, 1976.

[7] E. Dyer and R. Lashof, Homology of iterated loop spaces, Amer. J. Math., 84 (1962), 35-88.

[8] H. Hauschild, Zersplatung äquivarianter homotopiemengen, Math. Ann., 292 (1977).

[9] H. Hauschild, J. P. May, and S. Waner, Equivariant infinite loop space theory, (to appear).

[10] M. Laplaza, Coherence for distributivity, L.N.M. vol. 281, 29-65, Springer-Verlag, 1972.

[11] I. Madsen, On the action of the Dyer-Lashof algebra in $H_{*}(G)$, Pacific J. Math., 60 (1975), 235, 275.

[12] B. M. Mann and E. Y. Miller, The homology of the Burnside space, (preprint).

[13] B. M. Mann, E. Y. Miller and H. R. Miller, $S^{1}$-equivariant function spaces and characteristic classes, (to appear in T.A.M.S.).

[14] B. M. Mann and E. Y. Miller, On mapping tori and projective bundle constructions in PL cobordism, Indiana Un. Math. J., 31 (2) (1982), 255-272.

[15] J. P. May, Classifying spaces and fibrations, AMS Memoirs 155 (1975).

[16] _ With contributions by F. Quinn, N. Ray, and J. Tørnehäve, $E_{\infty}$ ring spaces and $E_{\infty}$ ring spectra, L.N.M. vol. 577, Springer-Verlag, 1977.

[17] _ Multiplicative infinite loop space theory, (to appear).

[18] R. J. Milgram, The mod 2 spherical characteristic classes, Ann. of Math. 92 (1970), 238-261.

[19] M. Nakaoka, Homology of the infinite symmetry group, Ann. of Math., 73 (1961), 229-257.

[20] S. Priddy, On $\Omega^{\infty} S^{\infty}$ and the infinite symmetric group, Alg. Top (Pro. Symp. Pure Math., vol. 22, Univ. Wisconsin, 1970), A.M.S., 217-220.

[21] D. Quillen, Higher algebraic K-theory I, L.N.M., vol. 341, 85-147, Springer-Verlag, 1973.

[22] R. Schultz, Equivariant function spaces and equivariant stable homotopy theory in Transformation Groups. ed. by Czes Kosniowski, Lon. Math. Soc. L.N.S., vol. 26 (1977), 169-189.

[23] _ Homotopy decompositions of equivariant function spaces I, Math. Z., 131 (1973), 49-75.

[24] _ Homotopy decomposition of equivariant function spaces II, Math. Z., 132 (1973), 69-90.

[25] G. B. Segal, Equivariant stable homotopy theory, Pro. International Congress of Mathematics, Nice 1970, vol. 2, 59-63.

[26] Configuration spaces and iterated loop-spaces, Inventiones Math., 21 (1973), 213-221.

[27] Some results in equivariant homotopy theory, (to appear).

[28] Categories and cohomology theories, Topology 13 (1974), 293-312.

[29] J. D. Stasheff, A classification theorem for fibre spaces, Topology, 2 (1963), 239-246.

[30] R. Steiner, A canonical operad pair, to appear in Math. Proc. Camb. Phil. Soc.

[31] R. E. Strong, Notes on Cobordism Theory, Princeton Univ. Press, 1968.

[32] R. Thomason and W. S. Wilson, Hopf rings in the bar spectral sequence, Quarterly Jorunal of Math., 31 (124) (1980), 507-511.

[33] A. Tsuchiya, Characteristic classes for spherical fibre spaces, Nagoya Math. J. 43 (1971), 1-39. 
[34] S. Waner, Equivariant homotopy theory and Milnor's theorem, Trans. Amer. Math. Soc., 258 (2) (1980), 351-368.

[35] _ Equivariant fibrations and transfers, Trans. Amer. Math. Soc., 258 (2) (1980), 369-384.

[36] B. M. Mann and E. Y. Miller, Mod 2 unstable equivariant spherical characteristic classes and Poincaré duality bordism generators, (preprint).

Received February 10, 1981 and in revised form, March 1982. Both authors were partially supported by the N. S. F. and the first named author was also partially supported by the Bowdoin College Faculty Research Fund.

BOWDOIN COLLEGE

BRUNSWICK, ME 04011

AND

PolyteChNic Institute of New York

BROOKLYN, NY 11201 
